\title{
The effect of inertia and flap on autorotation applied for hydrokinetic energy harvesting
}

\author{
Ali Bakhshandeh Rostami*, Antonio Carlos Fernandes \\ COPPE - Federal University of Rio de Janeiro \\ Corresponding author: bakhshandeh@oceanica.ufrj.br
}

\begin{abstract}
This paper outlines the results for a set of experiments carried out on Vertical Axis Autorotation Current Turbine (VAACT) which is an innovative concept among the other lowhead hydrokinetic energy converters. The VAACT is a one degree of freedom hinged plate which utilizes the autorotation phenomenon to rotate about its vertical axis in uniform water current. The state of the art on this turbine is application of the extra moment of inertia and also flapped shape of the rotor blade to improve the autorotation characteristics as the principle of operation of the turbine. Investigation of the effect of the extra moment of inertia and the flap on the operational characteristics such as maximum attainable angular velocity and tip speed ratio, obtainable torque of the turbine and its performance is the main objective of this paper.

The VAACT harvests energy efficiently from very low head current. The performance of the flapped configuration of this turbine reaches up to 33 percent in the very low current velocity. The highest efficiencies occur in the range of 0.65 to 0.85 of tip speed ratio and also in the range of 0.6 to 0.7 of the non-dimensional moment of inertia. Furthermore, this study has shown that the flap increases the power coefficient significantly by a factor of up to 5 compared to flat plate type.
\end{abstract}

Keywords:

Low head current, Autorotation Phenomenon, Hydrokinetic Energy Converter, Vertical Axis Autorotation Current Turbine (VAACT), Flapped plate type, Efficiency

Highlights:

- Flapped plate which is vertically hinged in mid chord shows autorotation motion.

- Extra moment of inertia on the turbine causes to improve the operation to harvest energy from the very low head water current.

- This simple model of the turbine harvests the energy with significant efficiency.

- Optimum range of moment of inertia has been discussed. 


\begin{tabular}{llll}
\multicolumn{2}{l}{ Nomenclature } & & \\
$\lambda$ & Tip Speed ratio $\left(=\omega C / 2 \mathrm{~V}_{\infty}\right)$ & $\rho$ & Fluid density $\left[\mathrm{Kg} / \mathrm{m}^{3}\right]$ \\
$\mathrm{I}^{*}$ & Dimensionless moment of inertia $\left(=\mathrm{I} / \rho \mathrm{C}^{5}\right)$ & $\omega$ & Turbine angular velocity $[\mathrm{Rad} / \mathrm{s}]$ \\
$\mathrm{Fr}$ & Channel Froude number $\left(=\mathrm{V}_{\infty} / \sqrt{g H}\right)$ & $\mathrm{I}$ & Mass moment of inertia $\left[\mathrm{kgm}{ }^{2}\right]$ \\
$\mathrm{Re}$ & Reynolds number $\left(=\rho \mathrm{V}_{\infty} C / \mu\right)$ & $\mathrm{f}_{\mathrm{V}}$ & Vortex Shedding Frequency $[\mathrm{Hz}]$ \\
$\mathrm{C}_{\mathrm{p}}$ & Power coefficient $\left(=\mathrm{P}_{\text {ext }} / 0.5 \rho \mathrm{HCV}_{\infty}{ }^{3}\right)$ & $\mathrm{f}_{\mathrm{r}}$ & Rotational Frequency [Hz] \\
$\eta$ & Efficiency $\left(=\mathrm{P}_{\text {taken }} / 0.5 \rho \mathrm{HCV}_{\infty}{ }^{3}\right)$ & $\mathrm{l}_{\mathrm{f}}$ & Chord length of flap [m] \\
$\mathrm{C}_{\mathrm{t}}$ & Torque Coefficient $\left(=\mathrm{T} / 0.5 \rho \mathrm{HC}^{2} \mathrm{~V}_{\infty}{ }^{2}\right)$ & $\gamma$ & Angle of inclination of flap [deg] \\
$\mathrm{St}$ & Strouhal number $\left(=f_{v} C / \mathrm{V}_{\infty}\right)$ & $\mathrm{m}$ & Weight of lifting object $[\mathrm{Kg}]$ \\
$\kappa$ & Chord ratio $\left(=\mathrm{l}_{\mathrm{f}} / \mathrm{C}\right)$ & $\mathrm{T}$ & Hydrodynamic torque $[\mathrm{N} . \mathrm{m}]$ \\
$\mathrm{Y}$ & Wetted surface aspect ratio $(=\mathrm{H} / \mathrm{C})$ & $\dot{\mathrm{h}}$ & Mean velocity of lifting the weight $[\mathrm{m} / \mathrm{s}]$ \\
$\mathrm{H}$ & Water depth [m] & $\omega_{\max }$ & Max. attainable angular velocity $[\mathrm{Rad} / \mathrm{s}]$ \\
$\mathrm{C}$ & Chord length of the $\mathrm{VAACT}[\mathrm{m}]$ & $\mathrm{g}$ & Gravitational acceleration $\left(=9.806 \mathrm{~m} / \mathrm{s}^{2}\right)$ \\
$\mathrm{V}_{\infty}$ & Current velocity $[\mathrm{m} / \mathrm{s}]$ & $\mathrm{C} p_{\max }$ & Max. power coefficient \\
$\mu$ & Dynamic viscosity $[\mathrm{kg} /(\mathrm{m} . \mathrm{s})]$ & $\mathrm{P}$ & Extractable power by turbine $[\mathrm{Watt}]$
\end{tabular}

\section{Introduction}

Wide sources of stream flow like tides, marine current, irrigation canal, and industrial flows can make a source of electricity generation by installing water turbine rotor. These devices should be capable to utilize the kinetic energy of these small water elevation streams. These technologies which are used for hydraulic head less than $2 \mathrm{~m}$ are often referred to as kinetic hydropower or hydrokinetic turbines [1]. Two main areas where hydrokinetic devices can be used in power generation purposes are (a) tidal current, and (b) river stream and also Ocean current.

The theoretical potential for ocean energy technologies has been estimated at 7,400 EJ/yr, well exceeding current and future human energy requirements [2]. The tidal stream energy extraction has been identified as an economically viable contributor to the renewable power. Rouker et al [3] have provided an up-to-date review of the status of tidal energy technology. Also, they have identified some of the key barriers challenging the development of tidal energy. In the other side, the rapid development of devices for tidal energy exploitation is being encouraged by government initiatives and by private investment [4]. Additionally, Güney and Kaygusuz [5] provide a review on the kinds of hydrokinetic turbines employed for river and tidal current, analyzing also the generator, control technologies and environmental impacts.

River current energy is a clean and renewable source of energy and has the advantage of predictability over other renewable energy sources. Based on one valuable study from 2007 in the USA to probe into the potential of the river current to generate the electricity, the current generating capacity of hydrokinetic energy in rivers and constructed waterways is $12800 \mathrm{MW}$ [6]. Then, free stream of the rivers can be used to generate the electricity by installing a suitable hydrokinetic turbine. Vermaak et al. [9] reviewed the state of the art of small scale micro hydrokinetic river technologies and found that the key barrier in the use of such technology in 
especially rural areas is the lack of research in the technical, economic and environmental benefits of such technologies.

Different designs of hydrokinetic converter as turbine and non-turbine type [1] are available for the extraction of kinetic energy of free stream flow, but the turbine type is the most used one [9]. The horizontal axis turbine is the most common design of a stream turbine, as shown in Figure 1, which comprises three categories of inclined axis, axial flow and transverse axis. A drawback with such designs is that their size cannot be increased significantly, because the limited depth of the flow at most sites restricts their diameter. Some researchers have been focused on efficient design of horizontal axis hydrokinetic turbine like [7], [8]. Anyi and Kirke [10] have reviewed works involving small axial flow hydrokinetic turbines specifically for generating electrical power for off-grid remote communities by river current. Moreover, the transverse horizontal axis water turbine (THAWT) has been studied by Mcadam et al [11] recently. Various configurations of the device were tested to assess the merits of varied blade pitch, rotor solidity, truss oriented blades and blockage ratio. Their study has demonstrated the ability of the device to exceed the Lanchester-Betz limit for kinetic efficiency by using high blockage.

In the other side, Gorlov, Darrieus, and Savonius turbines are vertical axis turbines as shown in Figure 2 . The Gorlov turbine [12] has helically shaped blades which are of more complicated nature of the blade's construction while the H-Darrieus turbine has straight blades, which are easy to be constructed but has some problems like self-starting resonant at a particular rotational speed. The Savonius turbine uses commonly a semi-circular section which can be constructed easily by a semi-circular pipe but has very low efficiency, so that Khan et al [13] have conducted some experimental measurements and found the maximum efficiency of Savonius is about 5 percent. The efficiency of a helical turbine for free flow condition as reported by Gorban et al. [14] is about $35 \%$ while this value is $23 \%$ for H-Darrieus type. Furthermore, Bachant and wosnik [15] have performed power measurements for cylindrical helical turbine (Gorlov) and spherical helical turbine (Lucid) as two different helical cross-flow marine hydrokinetic energy harvesters in a towing tank. They have reported the maximum efficiency of 35 percent for Gorlov (in $\lambda=2.3$ ) and 24 percent for Lucid (in $\lambda=2.2$ ) which $\lambda$ is tip speed ratio. 


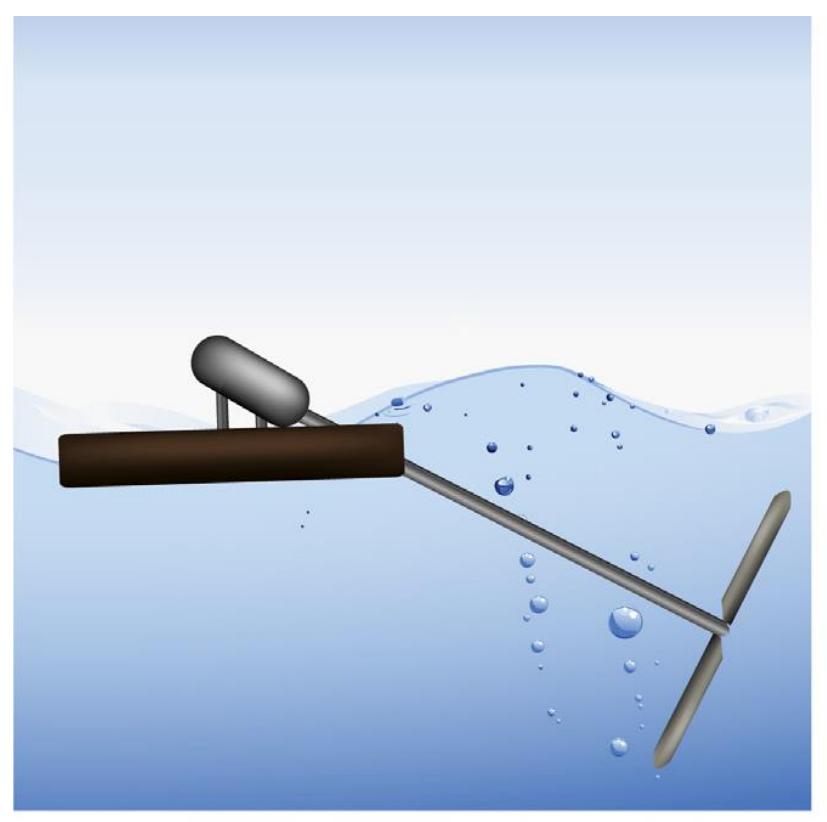

Inclined Axis

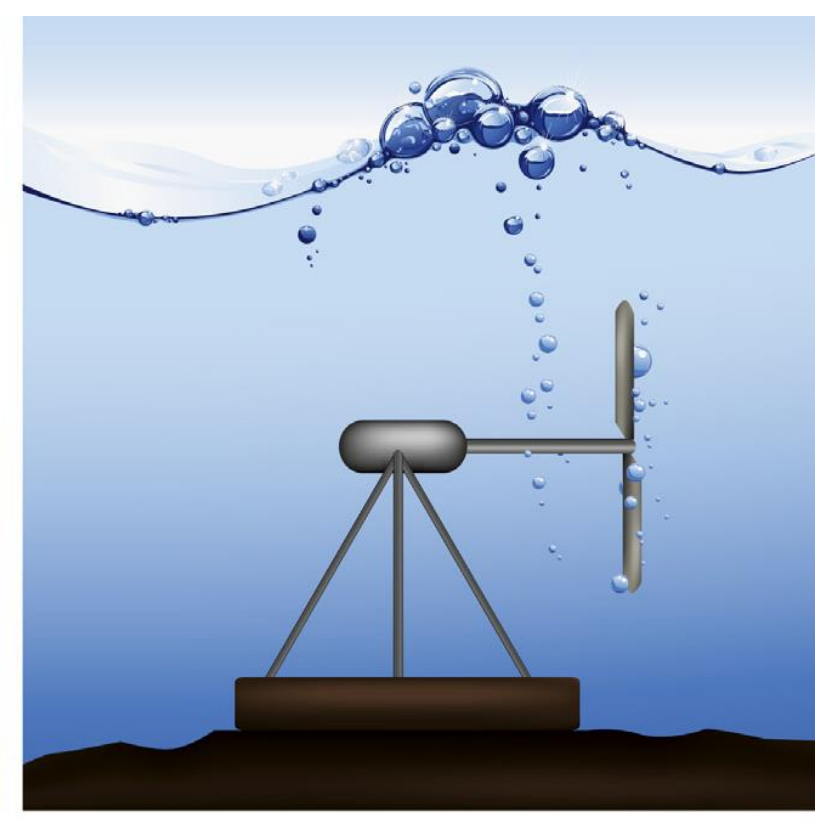

Axial Flow

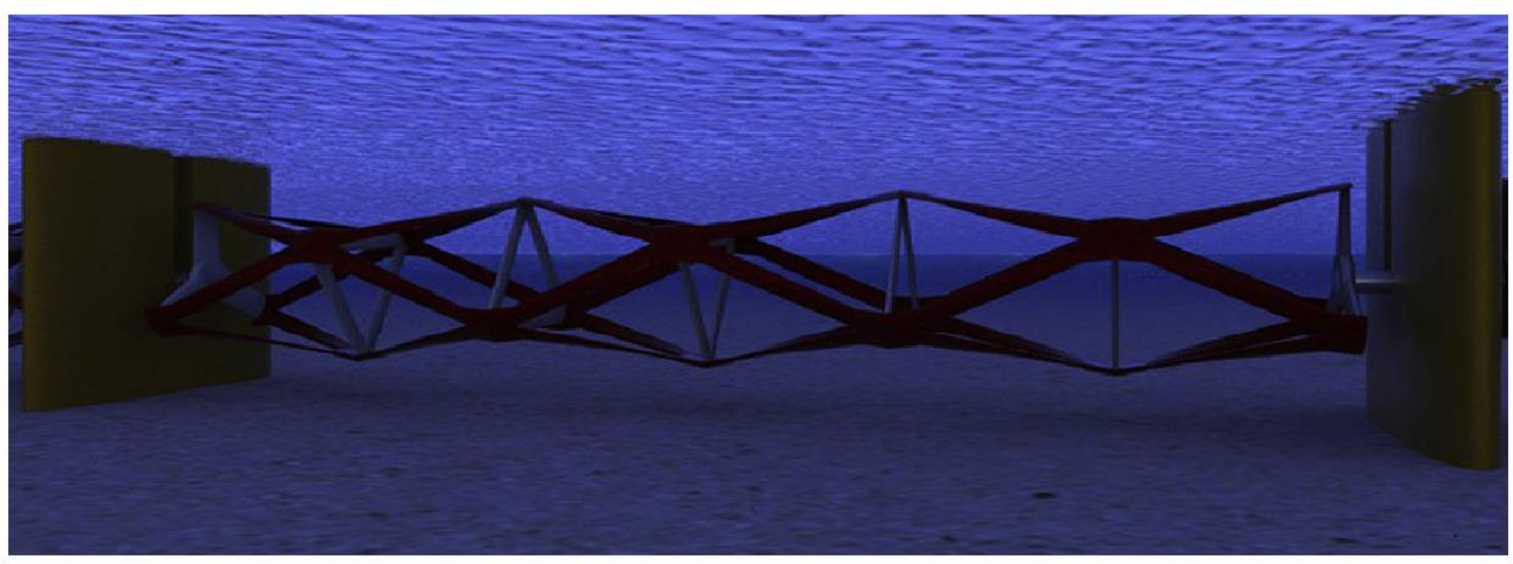

Transverse Axis

Figure 1: Horizontal axis turbine (photos are supplied by courtesy of $[9,11])$.

Hydrokinetic turbine technology has not been much utilized commercially yet because of some limitations such as high initial installation cost but it has got enormous potential to provide the power in low velocity condition. Due to the high cost of harnessing energy of water current by these turbines, it is very important to select a turbine that has an optimum performance at the selected site. In this way, Ramos and Iglesius [16] have presented a parametric approach to assess the performance of various water turbines like Gorlov helicoidal and Evapod turbines in their case study applied to a coastal bay of NW Spain. Also, Antheaume et al [17] have investigated numerically and experimentally the efficiency of one vertical axis water current turbine for free stream conditions versus farm conditions. They have concluded that the farm conditions cause an increase in efficiency. 


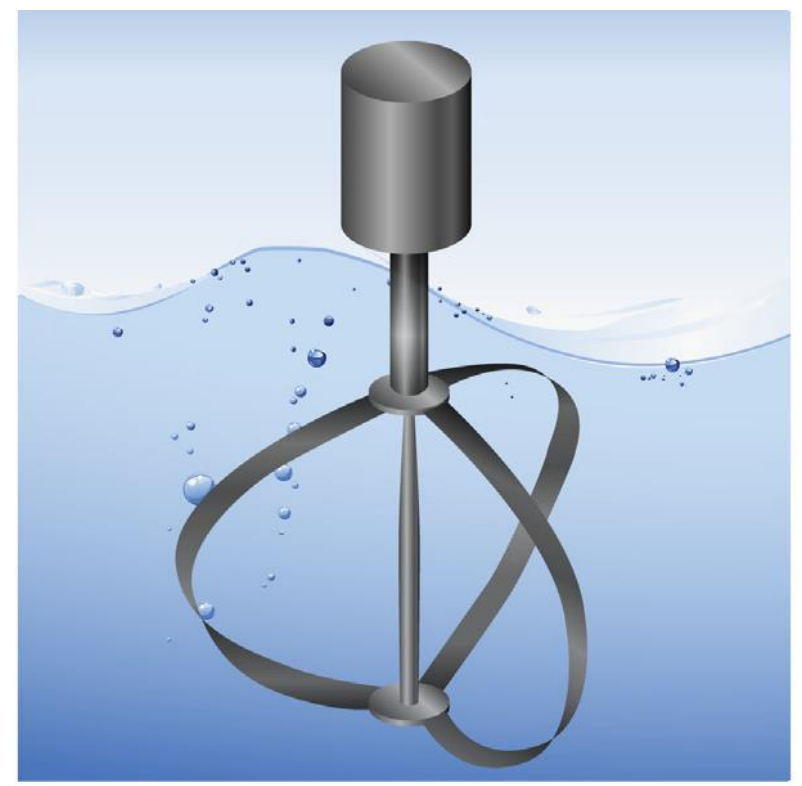

Darrieus

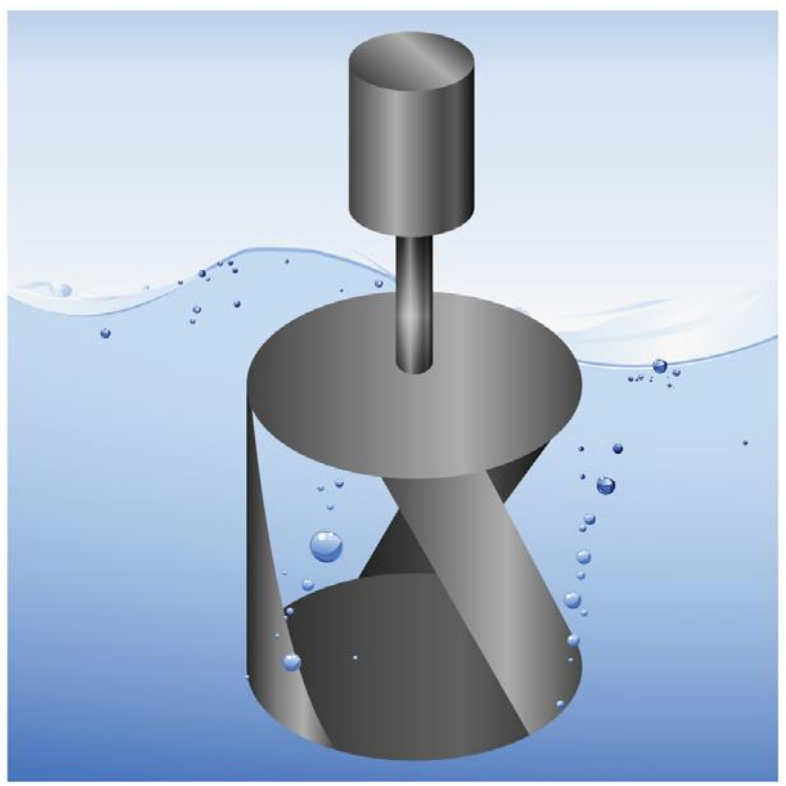

Gorlov

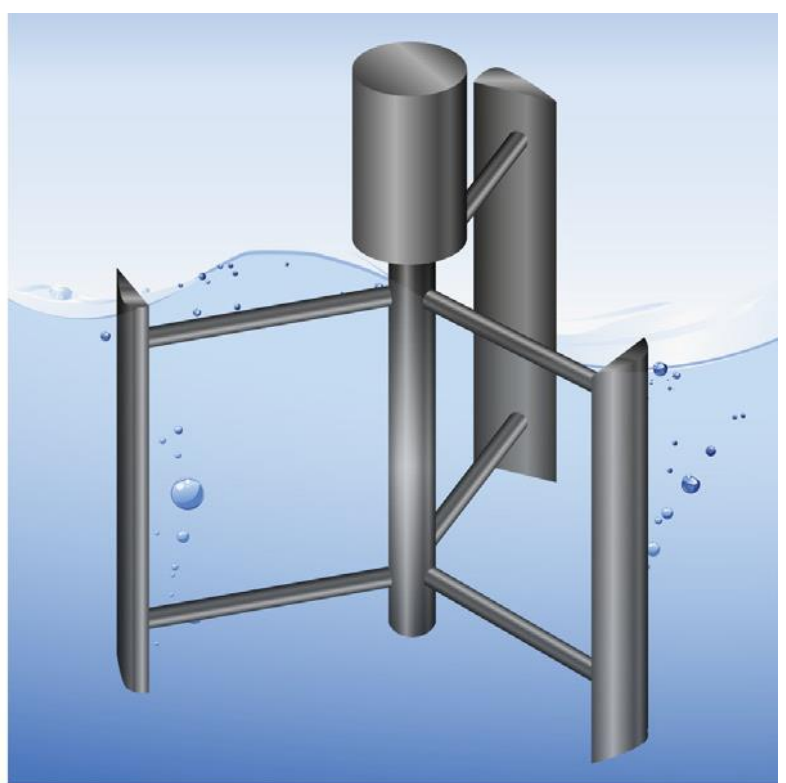

H- Darrieus

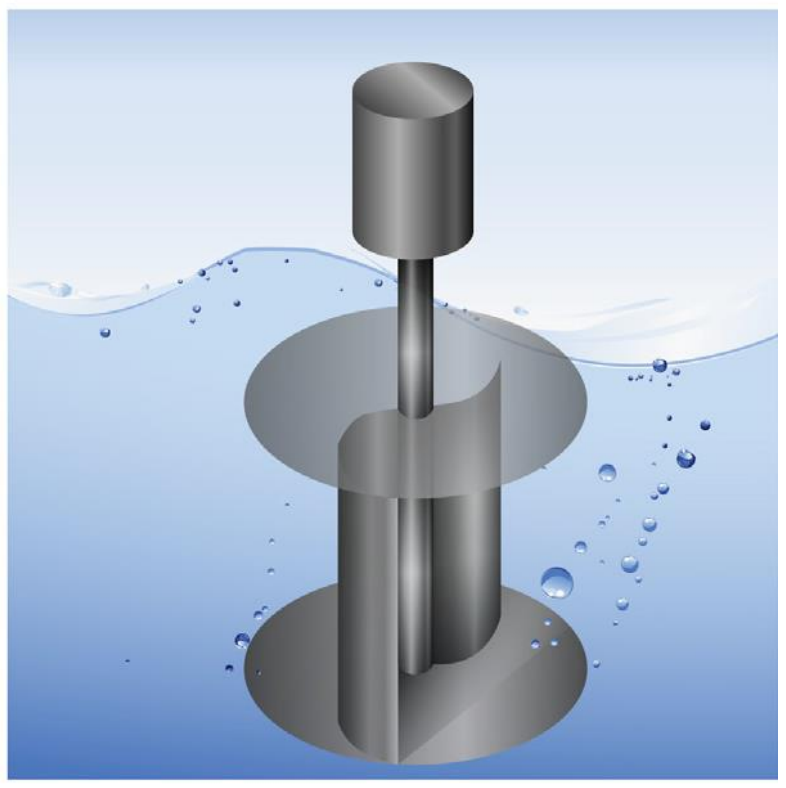

Savonius

Figure 2: Vertical axis turbine (photos are supplied by courtesy of [9]).

In the vertical axis domain, the Darrieus type turbines are common in the river energy applications [9]. Kirke [18] has conducted several tests in Australia and Canada on the Darrieus hydrokinetic turbine with fixed and variable pitch straight blades and fixed helical blades, with and without a slatted diffuser. He has considered the effect of mentioned parameters on the performance of turbine and found that the diffuser increases the power output by a factor of up to 3 compared to the same turbine without diffuser. Similarly, Ponta and Dutt [19] have made a study on the performance of a Darrieus type turbine through augmentation of the fluid flow 
incident on the turbine by incorporating a channeling device. As the same work, Malipeddi and Chatterjee [20] have carried out a computational study on the influence of duct geometry on the performance of the Darrieus type. They found that the duct geometry with straight external shape is superior when compared to a duct with convex external shape in respect of coefficient of power $(\mathrm{Cp})$.

One of the prominent turbines of the small scale current energy converters is the Savonius hydrokinetic turbine that has the capacity to self-start at a very low fluid velocity. To evaluate the hydrokinetic performance of the Savonius turbine at low velocity in an open water channel, Sarma et al [21] have conducted an experimental investigation along with computational fluid dynamic (CFD) study by using ANSYS 14.0. To understand the significance of the Savonius design in water application, the performance of the hydrokinetic turbine was experimentally compared to the identically designed wind turbine for the same input power values and was shown an enhanced performance of the former turbine. Kyozuka [22] reported an increase of the power coefficient of the Savonius turbine by combination of the Darrieus turbine. Based on his study, the attachment angle $90^{\circ}$ gave a better coefficient of the power when the turbine runs at the low speed or better starting torque. Also, Golecha et al. [23] have carried out an experimental study to optimize the position of the deflector plate at the upstream of the Savonius turbine in water current to maximize the power generated by the turbine. Furthermore, Moutsoglou and Weng [24] carried out experimental investigations to study the efficiency of Benesh rotor as an alternative for typical Savonius rotors giving low efficiency.

In addition, there are some more complicated configurations of the vertical axis turbine. For instance, Yang and Lawn [25] have paid attention to investigate a novel design of vertical axis turbine for tidal current, called the 'Hunter Turbine'. The turbine consists of several flapping blades that are hinged on a revolving drum. They found that the Hunter Turbine gives the satisfactory performance over a restricted range of flow with average power coefficient of 0.19 . Also, Hwang et al [26] have reported an investigation of a cycloidal water turbine. They have utilized an active control system to adopt the cycloidal straight blades according to the operating conditions to improve the turbine efficiency. Moreover, they have improved the self-starting characteristics of cycloidal turbine by the active control system.

As mentioned above, one class of hydrokinetic energy converters is non-turbine type which has oscillatory motion while rotational type is the other class that is named turbine type. As an example, such non-turbine device has been studied by Fernandes and Armandei [27] and [28] to extract hydrokinetic energy through torsional galloping phenomenon. In addition, Armandei et al [29] have published a feasibility assessment for energy harnessing by buffeting phenomenon. They have reported that the efficiency of buffeting based energy converter reaches to 60 percent, theoretically.

This paper considers studying a new type of vertical axis turbine which exploits the autorotation phenomenon as its principle of operation. This turbine is named Vertical Axis Autorotation Current Turbine (VAACT), and is a one degree of freedom hinged plate that rotates about its vertical axis in uniform water current. The state of the art on this turbine is application 
of extra mass moment of inertia to improve its operational characteristics in all the aspects such as to increase the rotational velocity and also enhancement of the performance. In the earlier configuration of this turbine, flat plate (simplest shape that can be manufactured) has been used as its rotating blade [30] and [31]. The present configuration is the developed one which has been equipped by two flaps. The idea of using flap, as mentioned in [32], has been arisen from high lift device theory in aerodynamic which is the second state of the art in this turbine.

This paper has an objective to probe into the effect of the extra mass moment of inertia and the flap on the operational characteristics like maximum attainable angular velocity and tip speed ratio, obtainable torque of the turbine and its performance. To understand the objective, tests have been conducted in the LOC at federal university of Rio do Janeiro (UFRJ). This paper presents and discusses the experimental result in different Reynolds numbers and nondimensional mass moment of inertias.

\section{Vertical Axis autorotation Current Turbine (VAACT)}

Autorotation phenomenon is the principle behind the operation of the VAACT. The phenomenon of autorotation is defined as continuous rotation of a freely rotatable body in uniform flow without external sources of supplied power. Therefore, an object with autorotation motion has a good potential to be considered as an energy harvester from the current [31]. Skews [33] has been probed into auto-rotational properties of bodies with different cross sections and reported that only bodies with less than eight sides are found to show auto-rotational behavior. Inasmuch as a plate which is hinged vertically on its axis of symmetry can rotate freely around the axis in the current, hence it has the potential to harvest energy of the flow as a turbine. This system is a one degree of freedom system which is called Vertical Axis Autorotation Current Turbine (VAACT). The VAACT has been introduced by Fernandes et al [30] and [31] with blade shape of flat plate. The earlier configuration is known as Flat Plate type [30]. Flapped Plate type or S shape type is the developed configuration of the VAACT which is shown in Figure 3. As can be seen from Figure 3, the VAACT consists of a vertically hinged plate in its mid chord and the extra mass to provide the extra moment of inertia.

The flapped profile shape is a flat plate with two end flaps, as shown in Figure 4, which are put in the opposite directions. The design of the present profile was inspired by the high lift devices approach in aerodynamic [34 and 35]. The flaps in S type cause to increase in lift and drag forces. Moreover, the flaps change the center of pressure on the VAACT and move it outward of the center. Consequently, this shape produces more yawing moment. 


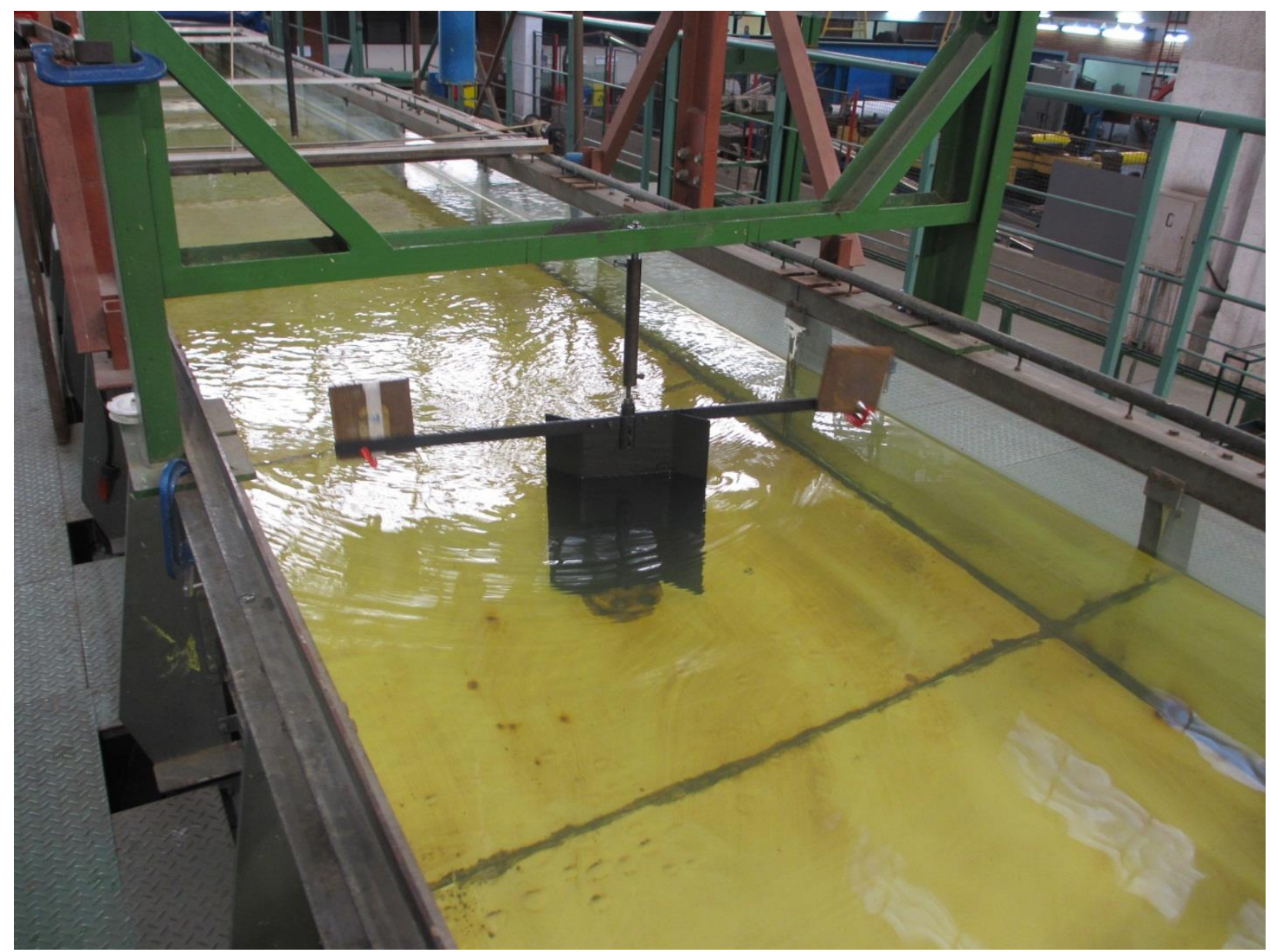

Figure 3: Closed up view of the VAACT (flapped shape type) in the LOC/current flume.

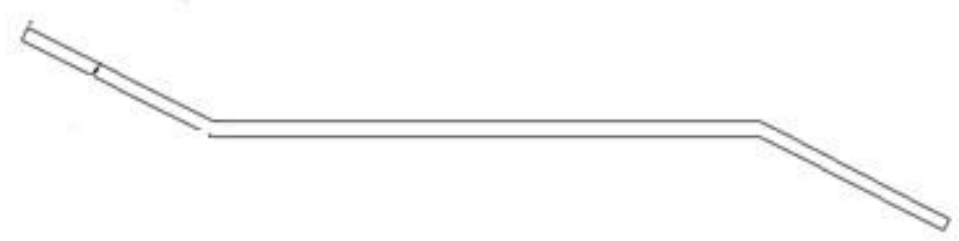

Figure 4: Schematic of VAACT (S shape type) in top view.

Principally, the exciting moment in auto-rotated object is produced by vortex formation behind the blade. In rotation of plate, a large vortex is shed from the retreating (downstream) face of the rotor, as shown in Figure 5, whereas no similar vortex is visible from the advance face. Consequently, such a vortex creates a hydrodynamic moment in direction of rotation which tends to continue autorotation. Moreover, as reported in some references [36, 37 and 38], the mass moment of inertia has to be sufficient to cause the autorotation phenomenon. For insufficient mass moment of inertia, the plate begins an oscillatory motion (Fluttering) around the perpendicular position to the current, whereas increase in the mass moment of inertia changes the 
fluttering mode to autorotation [39, 40 and 41]. The dimensionless moment of inertia is the ratio of mass moment of inertia of the turbine (I) to its added moment of inertia which shall be greater than 0.1 in the VAACT.
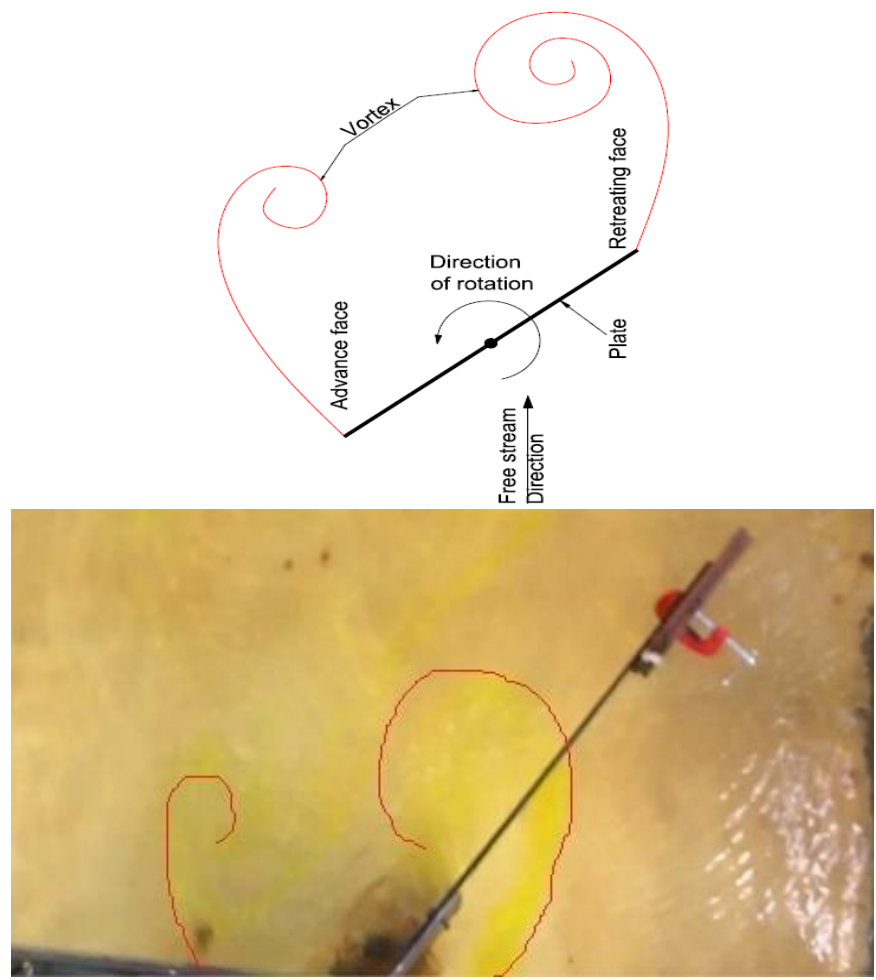

Figure 5: Vortex behind the VAACT observed in the LOC/current channel.

Therefore, continuous rotation in the VAACT demands two conditions simultaneously; appropriate exciting moment and enough mass moment of inertia of the system. The first condition is interpreted as adequate starting moment on the plate to overcome the inertia of rotation and sufficient moment to carry on the rotational motion. The exciting torque is applied by the hydrodynamic forces (lift and drag) on the blade. In each cycle of rotation there are four crucial positions (parallel and perpendicular to the current) wherein the hydrodynamic moment vanishes (lift and drag forces vanish). Therefore, sufficient mass moment of inertia (I) can help the turbine to cross over the critical situations [32].

\section{Methodology}

To measure the power coefficient of the turbine, a dimensional analysis was performed. Extractable power $\left(\mathrm{P}_{\text {ext }}\right)$ by the VAACT depends on eleven parameters which are summarized in Equation 1. Definition of each parameter has been given in Nomenclature.

$$
P_{\text {ext }}=f\left(g, \mu, \rho, C, \omega, V_{\infty}, H, I, f_{v}, l_{f}, \alpha\right)
$$

The dimensional analysis leads to 2 : 


$$
C_{p}=f\left(I^{*}, \lambda, R e, Y, S t, F r, \kappa, \alpha\right)
$$

where, $\mathrm{I}^{*}=\mathrm{I} / \rho \mathrm{HC}^{4}$ is the dimensionless moment of inertia, $\mathrm{Re}=\mathrm{V}_{\infty} \rho \mathrm{C} / \mu$ is the Reynolds number of the current, $\lambda=\omega \mathrm{C} / 2 \mathrm{~V}$ is the tip speed ratio of the turbine blade and $\mathrm{Y}=\mathrm{H} / \mathrm{C}$ is the wetted surface aspect ratio. The Strouhal number $\left(S t=f_{v} C / V_{\infty}\right)$ is a dimensionless parameter that describes vortex shedding. The Froude number, $F r=\mathrm{V}_{\infty} / \sqrt{g H}$, is a dimensionless value that describes the flow regimes in open channels. Also, $\kappa=l_{\mathrm{f}} / \mathrm{C}$ is the chord ratio which is the ratio of the flap chord to blade chord and relates to the flapped plate type.

In the laboratory, it was observed that two vortices are shed into the current in each cycle of rotation. Therefore, the frequency of vortex shedding $\left(f_{v}\right)$ is twice the rotational frequency $\left(f_{r}\right)$. Thus, the Strouhal number can be expressed as $S t=2 \lambda / \pi$ which $\lambda$ is the tip speed ratio. Then, the Strouhal number connects to the tip speed ratio and is eliminated the Eq. 2. Water depth was the same in all tests at 0.5 meter and then, all the tests have been done in a wetted surface aspect ratio of 1.66. As regards the constant environmental conditions during the experiments, Reynolds number and Froude number are congruence in the present experiments. In order to make a better sense about the environmental conditions, all diagrams are expressed in terms of Reynolds number in section 5. The angle of inclination $(\alpha)$ is zero for the flat plate type and 27 degrees for the flapped plate type. Finally, according to Eq. 3, the power coefficient $\left(\mathrm{C}_{\mathrm{p}}\right)$ is a function of non-dimensional mass moment of inertia $\left(I^{*}\right)$ and the tip speed ratio $(\lambda)$ and Reynolds number $(\mathrm{Re})$ :

$$
C_{p}=f\left(I^{*}, \lambda, R_{e}\right)
$$

Figure 8 shows a simple mechanical system which is used to calculate the efficiency of the turbine. One pulley is mounted on top of the axis of rotation that is connected to the suspended mass (m) by a nylon wire (see Figure 7, C). This pulley (pulley C) converts the turbine shaft rotation from vertical plane motion to horizontal plane motion. Other pulley (pulley B, see Figure 7, B) rearranges the horizontal plane motion into vertical direction to lift the mass. This weight shall be lifted $2.2 \mathrm{~m}$ vertically while the lifting time is being recorded. Thus, the extractable power is calculated by Eq. 4:

$$
P_{\text {ext }}=m g \dot{h}
$$

where $\dot{h}$ is the mean lifting velocity of the mass (m) up to 2.2 meters. Based on the background knowledge, the power coefficient $\left(C_{p}\right)$ or the efficiency $(\eta)$ is the ratio of the extractable power to the given power which is calculated by Eq. 5 :

$$
\eta=m g \dot{h} / 0.5 \rho H C V_{\infty}{ }^{3}
$$

Definitions and dimensions of different parameters in Eq. 5 have been given in Nomenclature. Also, the shaft torque was calculated by weight of mass which was lifted by nylon wire around the pulley, and rotational speed was measured by timing 10 revolutions. To calculate the 
efficiency, several sets of experiment were carried out. Each set of experiment includes one current velocity which is expressed in terms of Reynolds number, one dimensionless moment of inertia ( $\left.I^{*}\right)$ and one lifting mass. Table 1 shows the variety of different parameters for the sets of experiment. In order to check the repeatability, each set of experiment has been repeated three times.

Table 1: value of effective parameters in set of experiments for flat plate type

\begin{tabular}{||c||c||c||}
\hline \hline $\begin{array}{c}\text { Current Velocity } \\
(\mathrm{V} \infty)\end{array}$ & $\begin{array}{c}\text { Dimensionless moment of inertia } \\
\left(\mathrm{I}^{*}\right)\end{array}$ & $\begin{array}{c}\text { Lifting mass } \\
(\mathrm{m})\end{array}$ \\
\hline \hline $\begin{array}{c}\text { At least } 9 \text { different } \\
\text { values in range of } \\
0.068 \text { to } 0.271 \mathrm{~m} / \mathrm{s}\end{array}$ & $0.4,0.5,0.6,0.7,0.9$ & $\begin{array}{c}\text { At least } 8 \\
\text { different } \\
\text { weights }\end{array}$ \\
\hline
\end{tabular}

\section{Equipment setup}

\subsection{VAACT Model setup}

\subsubsection{Extra moment of inertia}

An extra mass is mounted on the VAACT to provide enough mass moment of inertia for the autorotation phenomena to occur. For this purpose, a unique apparatus has been constructed to increase the moment of inertia of the plate because in the water flume case, due to large water density compared to the air, increase in the moment of inertia is not straightforward. As shown in Figure 6, a lever arm is installed on top of the plate. Also, two masses of $4 \mathrm{Kg}$ are added to the system, which could be placed in different positions of the bar to supply it with a specific moment of inertia.

\subsubsection{Blade width}

An object placed in a channel will experience different forces compared with the same object placed in an unrestricted flow. This effect is known as blockage [42] and is reasonably well understood for wind tunnel and towing tank experiments. In the channel, a plate with large width experiences high blockage effect [43]. Therefore the maximum breadth of the plate shall be optimized for each channel. Maskel [42] has pointed out that the suitable blockage effect for bluff body in the channel is less than 0.2 . Hence, a plate with $0.3 \mathrm{~m}$ in width was selected which the blockage effect is 0.21 . The important characteristics of the VAACT are listed in Table 2. 


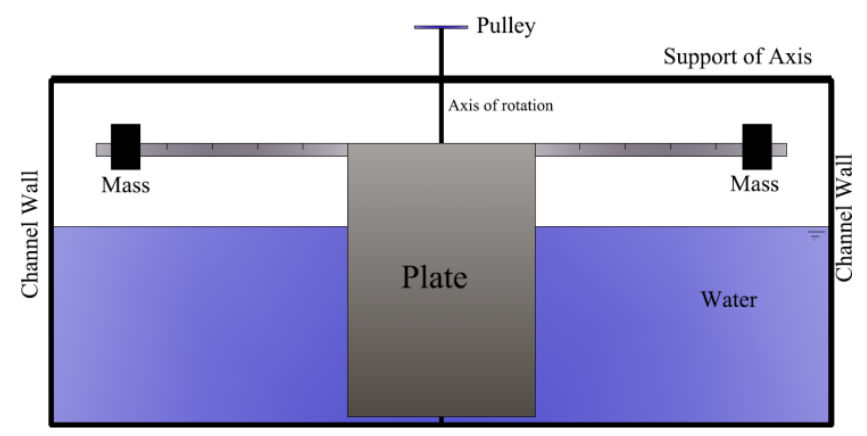

Figure 6: The experimental setup to provide different mass moment of inertia for VAACT in front view.

Table 2: Characteristics of the VAACT blade

\begin{tabular}{ll}
\hline \multicolumn{2}{c}{ Blade properties } \\
\hline Materials & Aluminum \\
Thickness & $5 \mathrm{~mm}$ \\
Height & $0.6 \mathrm{~m}$ \\
Total width for Flapped plate & $0.34 \mathrm{~m}$ \\
Width projected on perpendicular of & $0.3 \mathrm{~m}$ \\
current direction & 1.66 \\
Wetted aspect ratio & 0.21 \\
\hline Blockage ratio & \\
\hline
\end{tabular}

\subsection{Performance measurement setup}

A special setup has been designed to conduct the experiments to measure the performance of the VAACT in the current channel (22 $\mathrm{m}$ in length, $1.4 \mathrm{~m}$ in width and $0.75 \mathrm{~m}$ in depth) at LOC, as shown schematically in Figure 8 . A uniform water current with maximum velocity of $0.5 \mathrm{~m} / \mathrm{s}$ was circulated using four centrifugal pumps. The flow rate was regulated by changing the rotational velocity of pumps. The water depth can be controlled separately by changing the height of a sluice gate at the downstream end but in the present experiments, water depth was set to $0.5 \mathrm{~m}$. Thus, the maximum Froude number $\left(V_{\infty} / \sqrt{g H}\right)$ of the channel is 0.22 (correspond to velocity of $0.5 \mathrm{~m} / \mathrm{s}$ ), which indicates the current state of the channel is subcritical or tranquil flow.

Furthermore, to reduce the level of turbulence of the current, a honeycomb has been installed after pump nozzle. The velocity of the water current was measured by a propeller type current meter (see Figure 7 A). Current meter was placed at a distance of $6 \mathrm{~m}$ far from the honeycomb and the turbine was put $5 \mathrm{~m}$ away the current meter, in the mid length of the channel. For assurance, the current velocity was recorded during the experiments in each Reynolds number. Average value of the recorded velocity was considered to calculate the Reynolds number. For various velocity measurements of the carried out experiments, the standard deviation of recorded velocities were less than 5 percent of mean ones. The uncertainty of the measured velocity is less than 1 percent. The environmental characteristics of the experiments are shown in Table 3. 


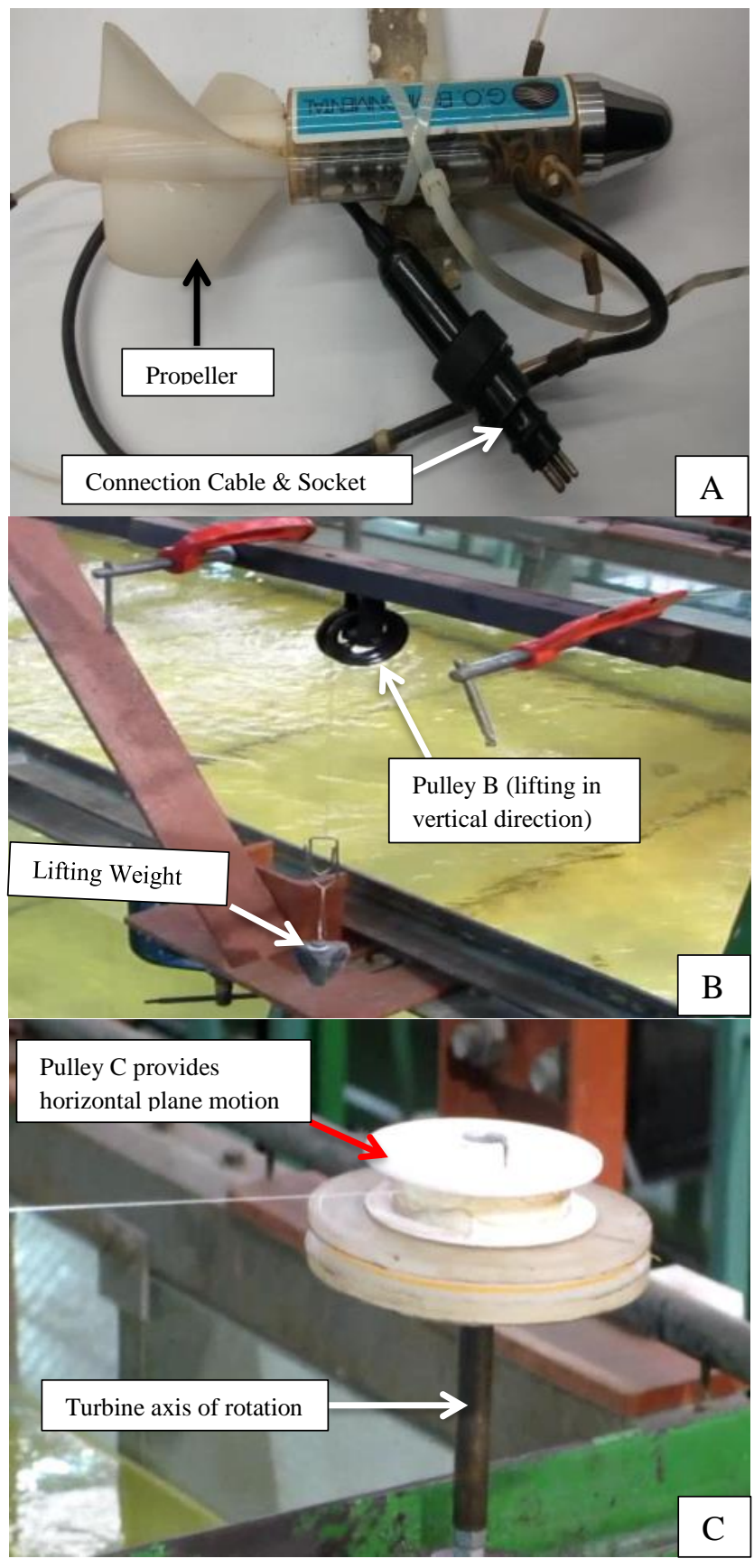


Figure 7: different component of experimental setup to measure the efficiency (A: current meter, B and C: pulley B arranges the lifting in vertical direction and pulley $\mathrm{C}$ creates horizontal plane motion).

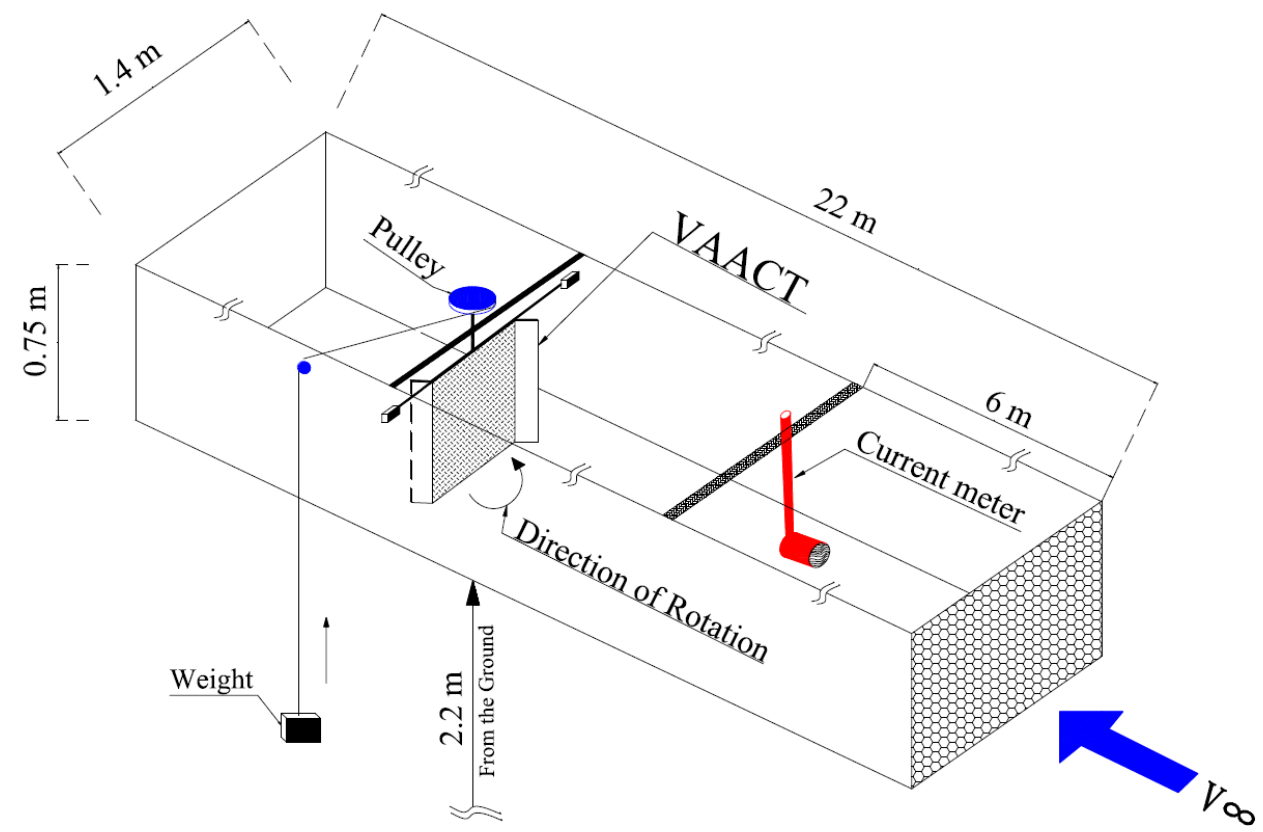

Figure 8: Schematic setup for experiment of VAACT in the current channel of LOC/COPPE/UFRJ.

Table 3: The environmental characteristics of experiments

\begin{tabular}{lc}
\hline \multicolumn{2}{c}{ Fluid properties } \\
\hline Fluid type & Fresh water \\
Fluid density $(\rho)$ & $1000 \mathrm{~kg} / \mathrm{m}^{3}$ \\
Current velocity $\left(\mathbf{V}_{\infty}\right)$ & $0.068-0.271 \mathrm{~m} / \mathrm{s}$ \\
Flow Reynolds number $(\mathrm{Re})$ & $20000-82000$ \\
Environmental temperature & $20^{\circ}$ \\
Current depth $(\mathrm{H})$ & $0.5 \mathrm{~m}$ \\
Channel Froude number $(\mathrm{Fr})$ & $0.031-0.122$ \\
\hline
\end{tabular}

\section{Result and discussion}

In this section, the figures are prepared based on the results of the experiments conducted in different current velocities in the range of 0.068 to $0.271 \mathrm{~m} / \mathrm{s}$. Discussion about the experimental data covers three distinct subjects, i.e. the maximum angular velocity and the highest attainable tip speed ratio of the turbine, the extractable torque and also the efficiency. In each case, the effect of the Reynolds number (Re) and the non-dimensional moment of inertia (I*) and also the flap will be assessed.

\subsection{Maximum attainable rotational kinematic}

The maximum attainable rotational kinematic includes the maximum angular velocity and the tip speed ratio of the VAACT. These characteristics are related to the unloading condition 
wherein the turbine is rotating without lifting the weight. Figure 9 shows the experimental results of the maximum angular velocity $\left(\omega_{\max }\right)$ of the flapped plate type of the VAACT. As it can be seen, this turbine operates in low rotational speed which can be considered as fish benign. In other words, fish can recognize the turbine as an obstacle and escape unhurt. Figure 9 reveals that there has been a gradual rise in the amount of angular velocity by increasing the nondimensional moment of inertia $\left(I^{*}\right)$. In other words, in each Reynolds number, the value of the optimum I* correspond to the highest amount of the angular velocity is greater than 0.9 (the highest value of tested $\left.I^{*}\right)$.

It is so clear that increase in the Reynolds number causes to increase in the angular velocity, while the tip speed ratio goes down. From Figure 10, the optimum value of $I^{*}$ for the tip speed ratio places out of the tested range of this parameter because, $\mathrm{I}^{*}$ that is increased, the achievable tip speed ratio is being risen.

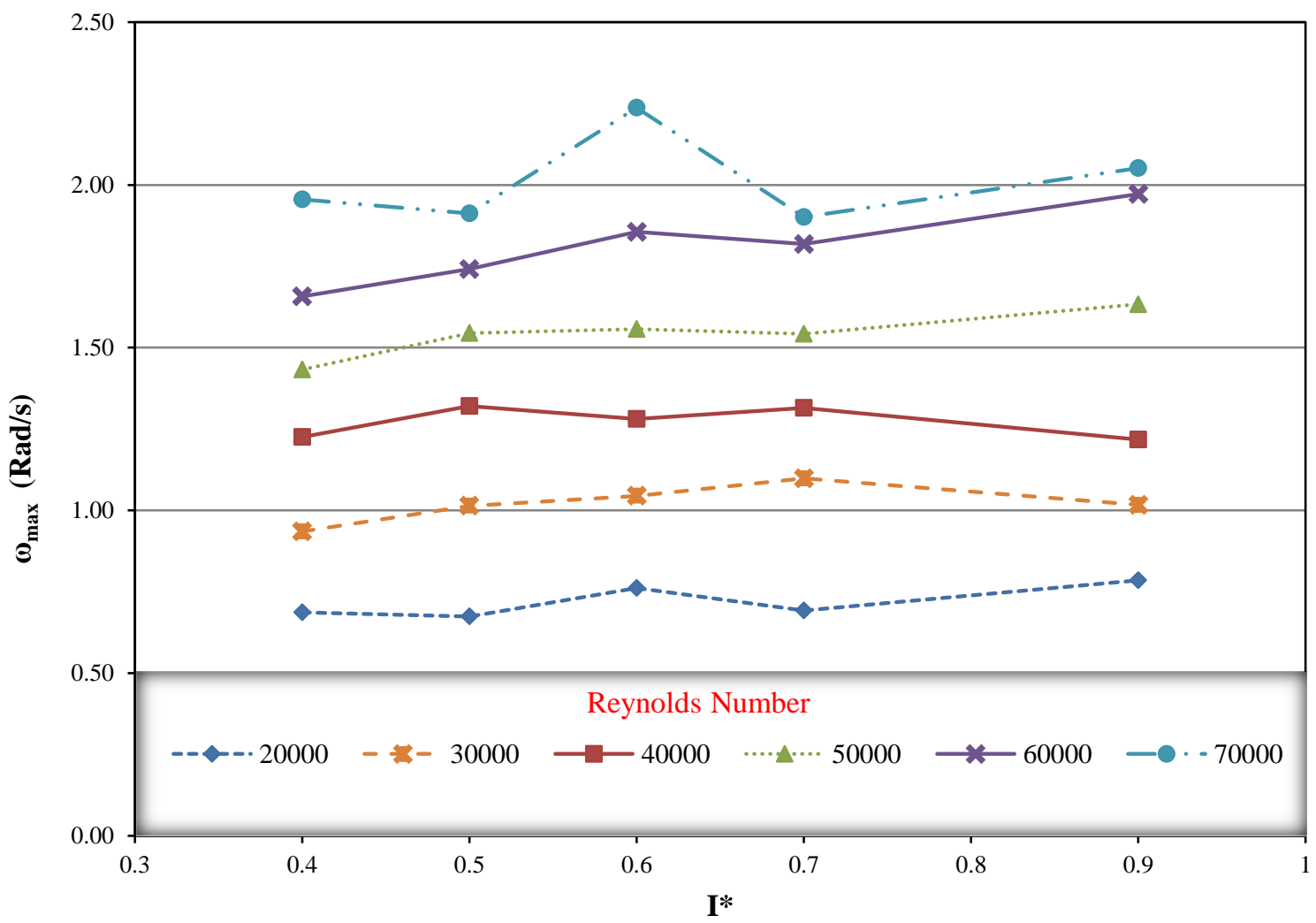

Figure 9: Maximum achievable angular velocity of flapped plate type of VAACT in various Reynolds number with respect to I* (non-dimensional mass moment of inertia). 


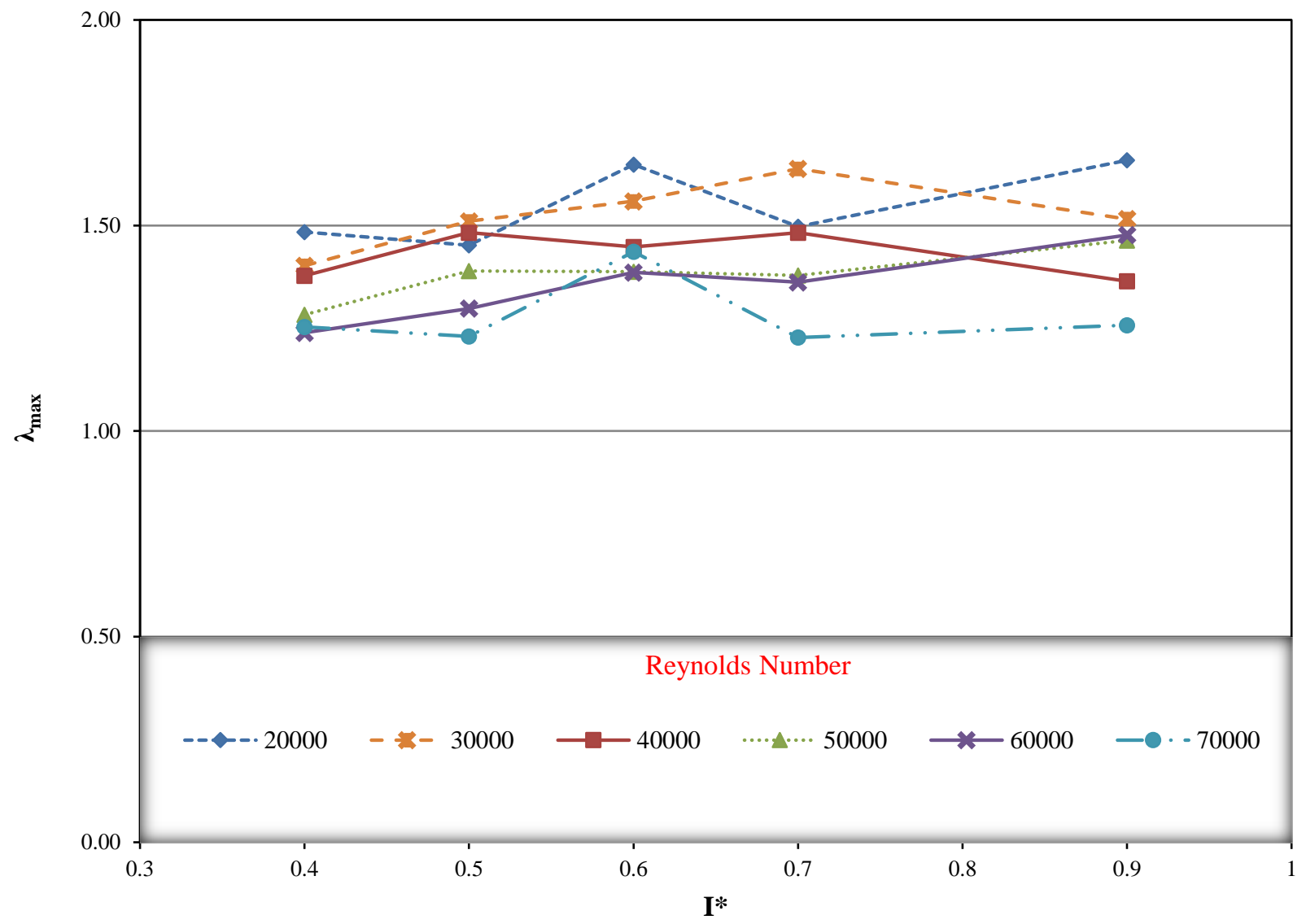

Figure 10: Highest tip speed ratio $(\lambda \max )$ can be obtained by flapped plate type of VAACT in various Reynolds number with respect to $I^{*}$ (non-dimensional mass moment of inertia).

A proper evaluation of the effect of the flap on the highest achievable angular velocity $\left(\omega_{\max }\right)$ and the tip speed ratio $\left(\lambda_{\max }\right)$ in comparison with the flat plate type of the VAACT should be carried out in the same I* and Reynolds number. Hence, Table 4 and Table 5 have presented their values in four Res and I*s. These tables reveal that there is a significant increase in the value of $\omega_{\max }$ and $\lambda_{\max }$ due the flap. As can be observed from Table 6 , the flap can increase the angular velocity of the VAACT averagely 60 percent however this percentage is dependent on the value of $I^{*}$ and Re. For example in $I^{*}=0.9$, the flap increases the $\omega_{\max }$ of the turbine more than twice in comparison with the flat plate type. These values for $\lambda_{\max }$ are similar to those mentioned for $\omega_{\max }$.

Table 4: Value of $\omega_{\max }$ for Flat plate and $\mathrm{S}$ shape type in different Reynolds number and I*

\begin{tabular}{c|cc|cc|ccc||cc}
\hline \multicolumn{1}{|l|}{ Re } & \multicolumn{2}{|c|}{40000} & \multicolumn{2}{c|}{50000} & \multicolumn{2}{c}{60000} & \multicolumn{2}{c}{70000} \\
\hline I* & Flat & S & Flat & S & Flat & S & Flat & S \\
\hline \hline 0.4 & 0.736 & 1.225 & 0.937 & 1.432 & 1.137 & 1.657 & 1.281 & 1.955 \\
0.5 & 0.959 & 1.320 & 1.077 & 1.545 & 1.203 & 1.741 & 1.580 & 1.913 \\
0.7 & 0.790 & 1.315 & 0.984 & 1.542 & 1.151 & 1.818 & 1.287 & 1.901 \\
0.9 & 0.561 & 1.217 & 0.788 & 1.633 & 1.048 & 1.972 & 1.234 & 2.052 \\
\hline \hline
\end{tabular}


Table 5: Value of $\lambda_{\max }$ for Flat plate and S shape type in different Reynolds number and I*

\begin{tabular}{c||cc||cc||cc||cc}
\hline \multicolumn{1}{|l|}{ Re } & \multicolumn{2}{|c|}{40000} & \multicolumn{2}{c|}{50000} & \multicolumn{2}{c}{60000} & \multicolumn{2}{c}{70000} \\
I* & Flat & S & Flat & S & Flat & S & Flat & S \\
\hline \hline 0.4 & 0.817 & 1.378 & 0.834 & 1.283 & 0.849 & 1.239 & 0.822 & 1.254 \\
0.5 & 1.074 & 1.483 & 0.983 & 1.390 & 0.898 & 1.298 & 1.015 & 1.230 \\
0.7 & 0.854 & 1.482 & 0.882 & 1.379 & 0.859 & 1.362 & 0.824 & 1.228 \\
0.9 & 0.614 & 1.364 & 0.703 & 1.464 & 0.778 & 1.477 & 0.790 & 1.258 \\
\hline \hline
\end{tabular}

Table 6: Effect of flap on $\lambda \max$ and $\omega \max$ (value is presented in percent)

\begin{tabular}{c|cccc}
\hline I* & $\mathbf{4 0 0 0 0}$ & $\mathbf{5 0 0 0 0}$ & $\mathbf{6 0 0 0 0}$ & $\mathbf{7 0 0 0 0}$ \\
\hline \hline $\mathbf{0 . 4}$ & 69 & 54 & 46 & 53 \\
$\mathbf{0 . 5}$ & 38 & 41 & 45 & 21 \\
$\mathbf{0 . 7}$ & 74 & 56 & 59 & 49 \\
$\mathbf{0 . 9}$ & 122 & 108 & 90 & 59 \\
\hline \hline
\end{tabular}

\subsection{Achievable Torque}

Assessment of the effect of I* and Re on the obtainable torque can be carried out by Figure 11 to Figure 13. These figures consist of experimental data of the seven different Reynolds numbers in three values of the tip speed ratios, i.e. $0.65,0.75$ and 0.85 .

Any increase in Reynolds number leads to decrease in the available torque coefficient as a result of the dissipation of energy by the vortex shedding that is increased in higher angular velocities. It can be inferred from the figures in this section that the lower Reynolds number is more appropriate to attain the higher torque coefficient for this turbine because the higher Reynolds number decreases the torque coefficient significantly.

According to the angular momentum theory, the non-hydrodynamic torque is added to the VAACT system due to the extra mounted mass. This torque (in the rotational rigid body) is proportional to the mass moment of inertia and the angular acceleration as follow:

$$
\tau_{n e t}=I . d^{2} \beta_{s} / d t^{2}
$$

Based on the momentum theory, for a constant given energy to the system, increase in the mass moment of inertia causes to decrease in the angular acceleration. Hence, in a constant current velocity, higher mass moments of inertia decrease the angular acceleration. Thus, the total torque coefficient descends in higher mass moments of inertia. Also, the attainable torque of the VAACT will decrease in lower mass moments of inertia. Therefore, in special range of $I^{*}$, the additional torque helps to increases the total moment of the system. In other words, the dimensionless moment of inertia has an optimum value near the 0.6 for the flapped plate 
configuration. This value is reliable for the entire tip speed ratios and Reynolds numbers approximately.

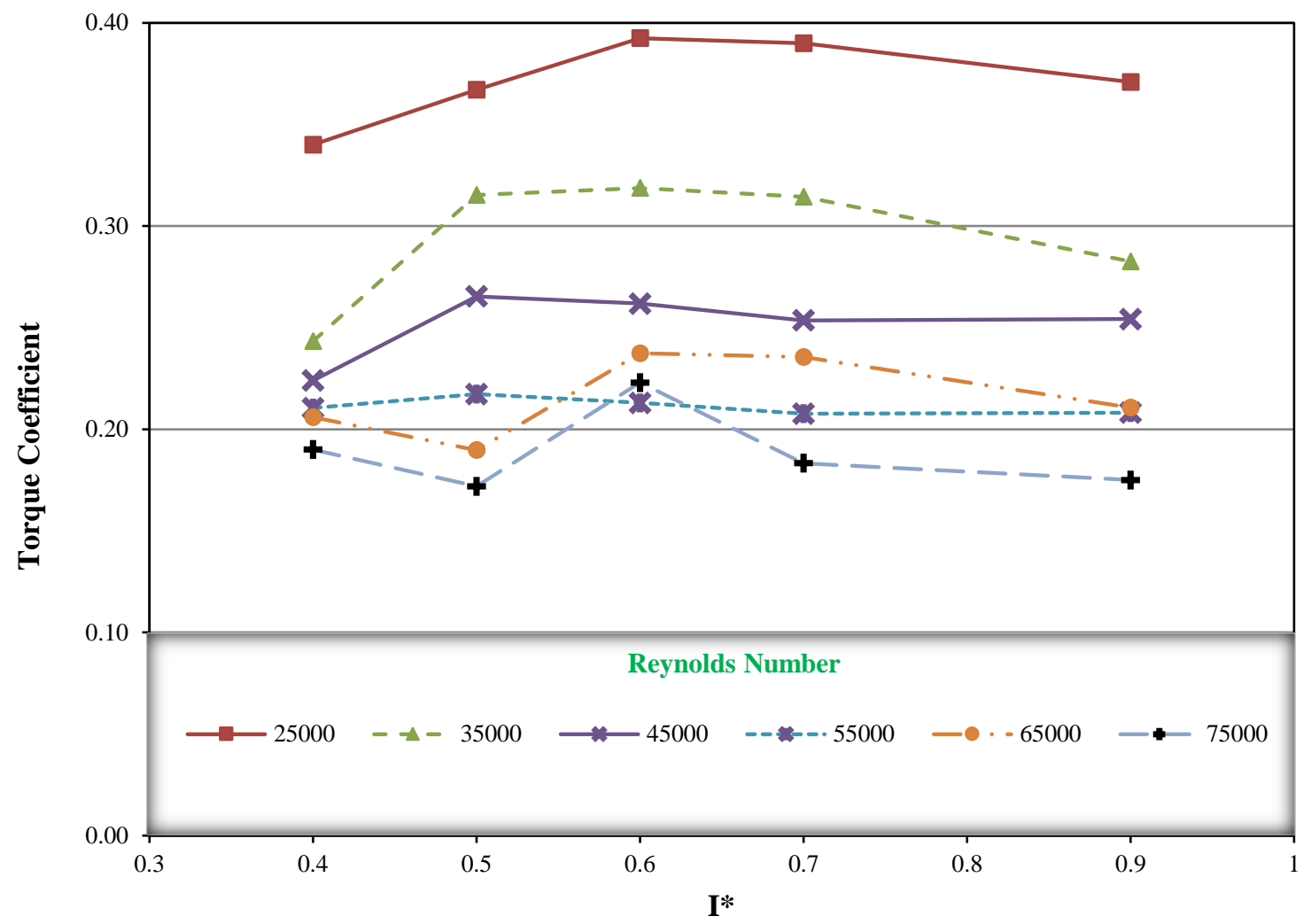

Figure 11: Torque coefficient with respect to I* in different Reynolds number and $\lambda=0.65$ for flapped plate type. 


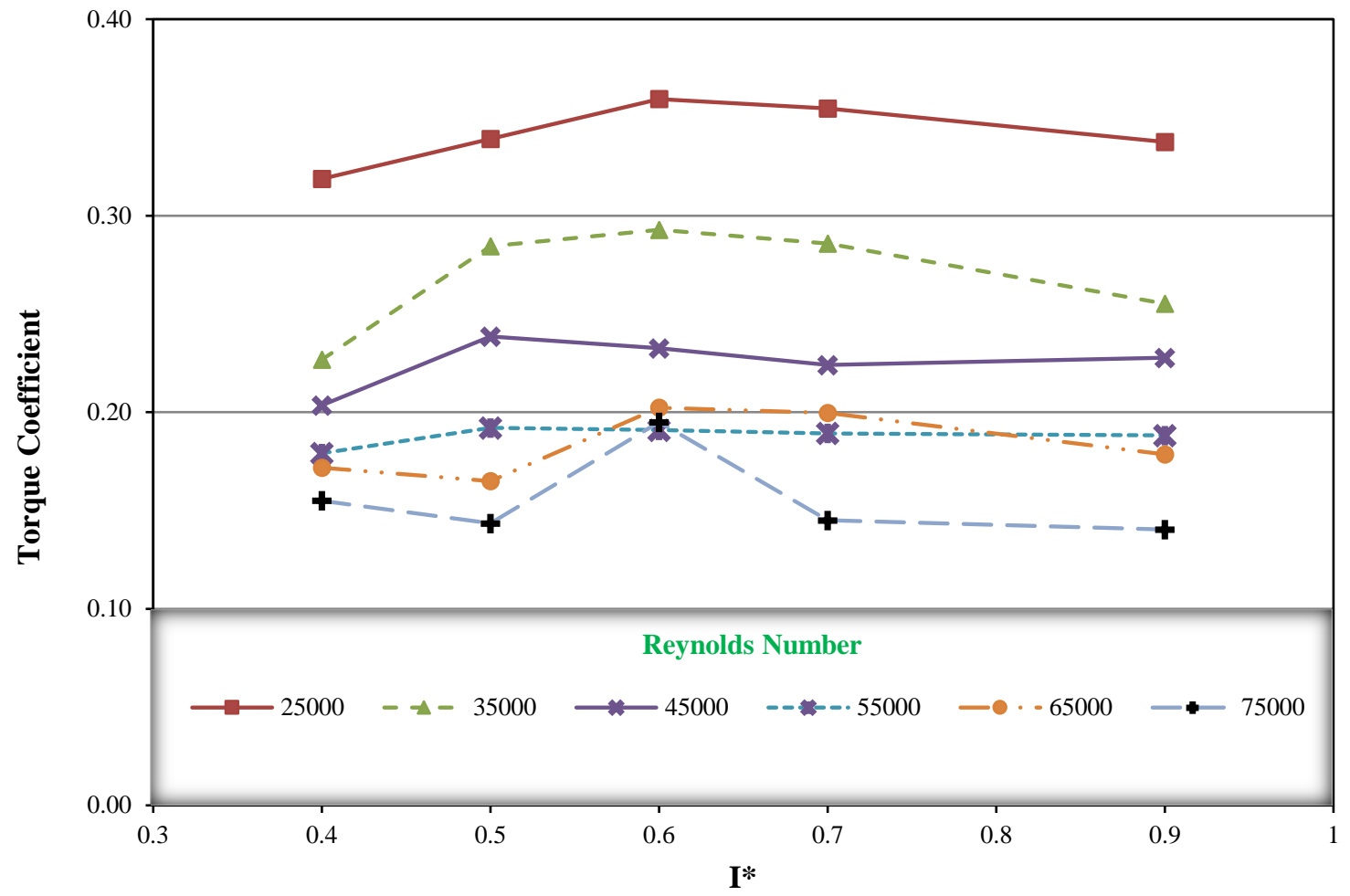

Figure 12: Torque coefficient with respect to I* in different Reynolds number and $\lambda=0.7$ for flapped plate type.

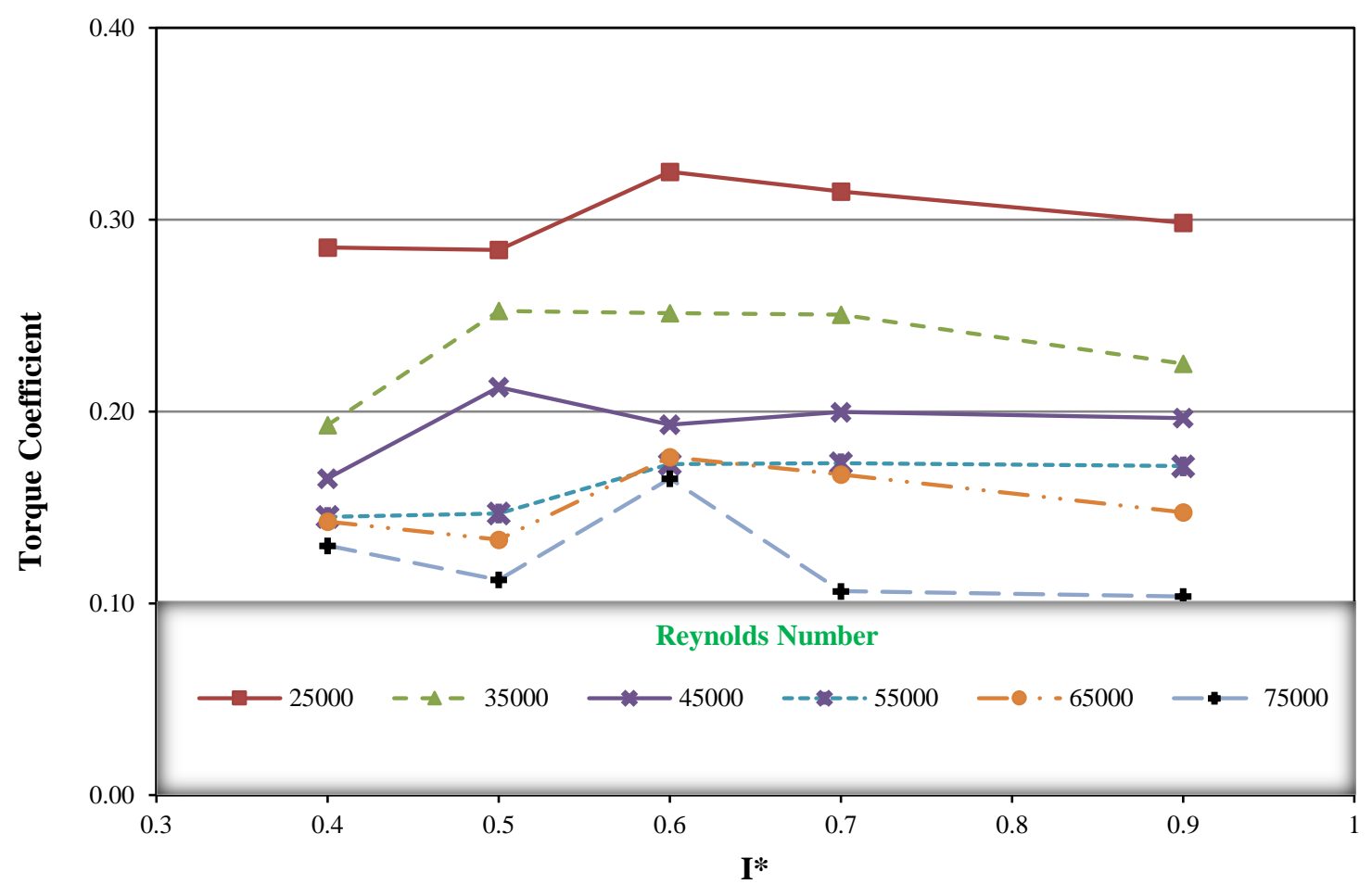

Figure 13: Torque coefficient with respect to $I^{*}$ in different Reynolds number and $\lambda=0.85$ for flapped plate type. 


\subsection{Efficiency data}

The power coefficient of the flapped plate turbine is given in Figure 14 to Figure 18 against the tip speed ratio in different Reynolds numbers and different dimensionless mass moments of inertia.

According to the experimental data, increase in the tip speed ratio of the turbine causes the efficiency to descend. The reason can be interpreted as increase in the energy dissipation as explained in the forgoing section. Then, the optimum value of the tip speed ratio correspond to the highest efficiency exists for all the Reynolds numbers. It can be seen from Figure 14 to Figure 18 that the optimum tip speed ratio correspond to the highest efficiency places in the range of 0.65 to 0.85 in each Reynolds number.

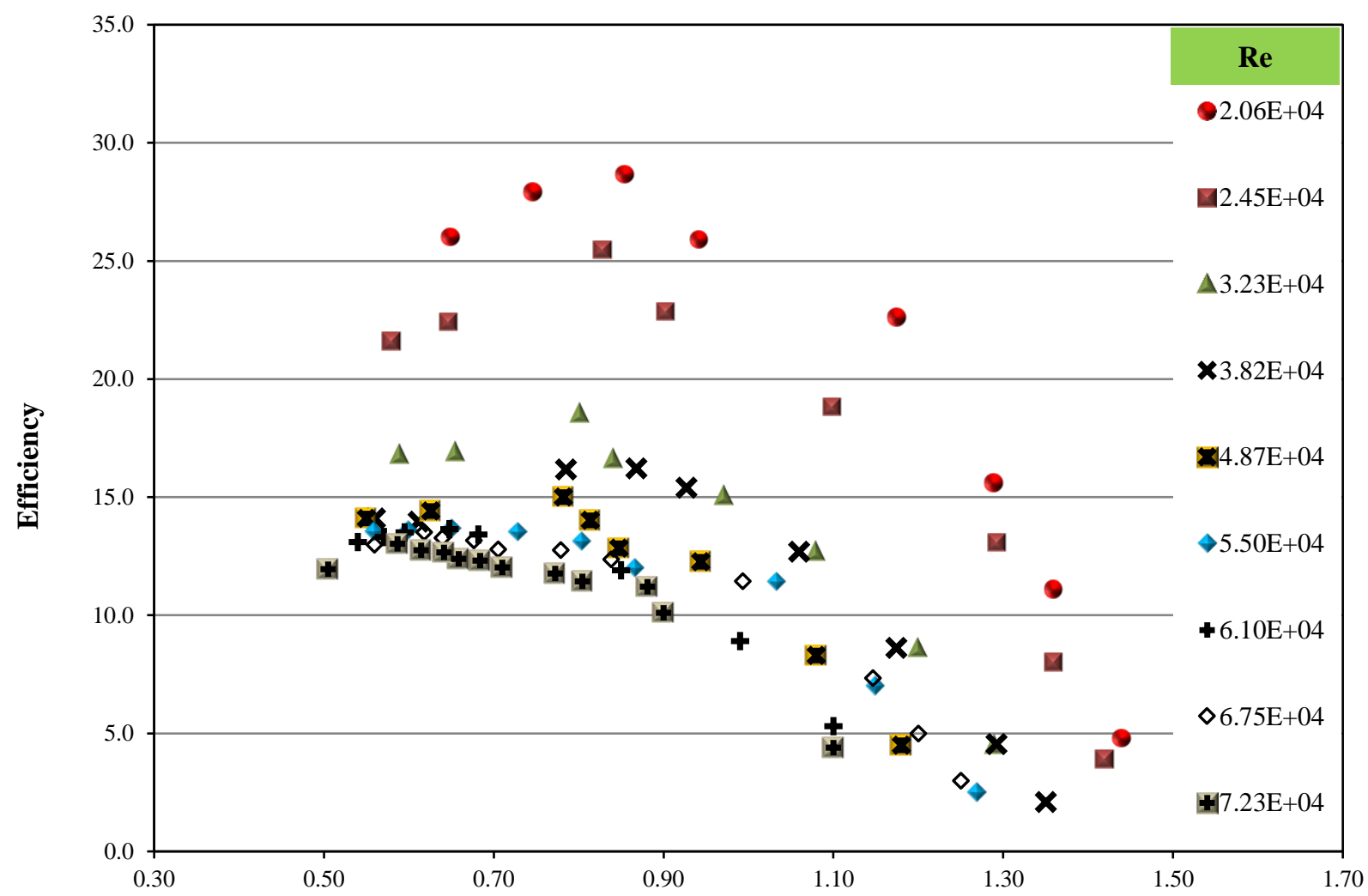

Tip Speed Ratio

Figure 14: Experimental data for efficiency of the VAACT (Flapped Type) in different Reynolds number (I*=0.4). 


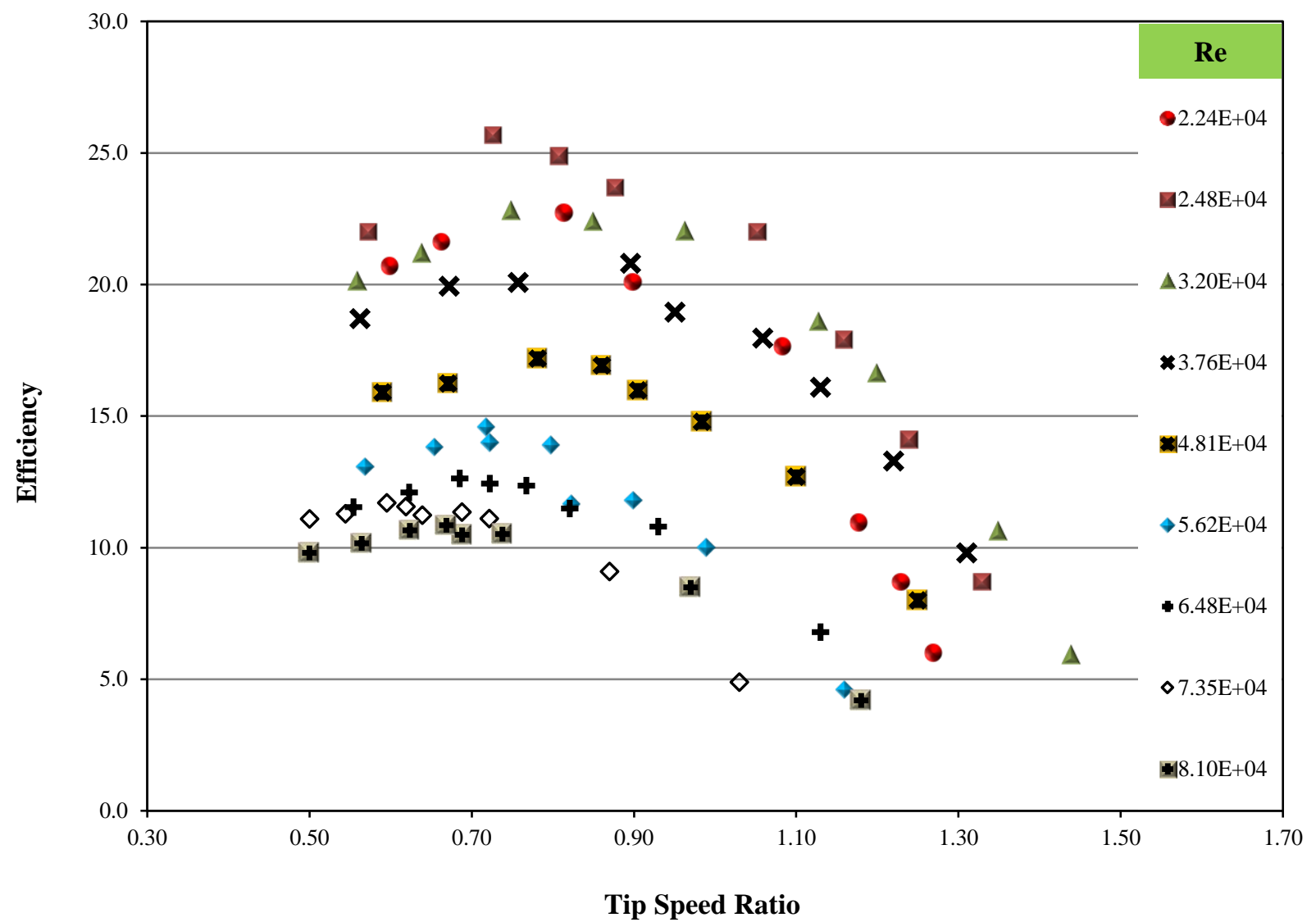

Figure 15: Experimental data for efficiency of the VAACT (Flapped Type) in different Reynolds number (I*=0.5). 


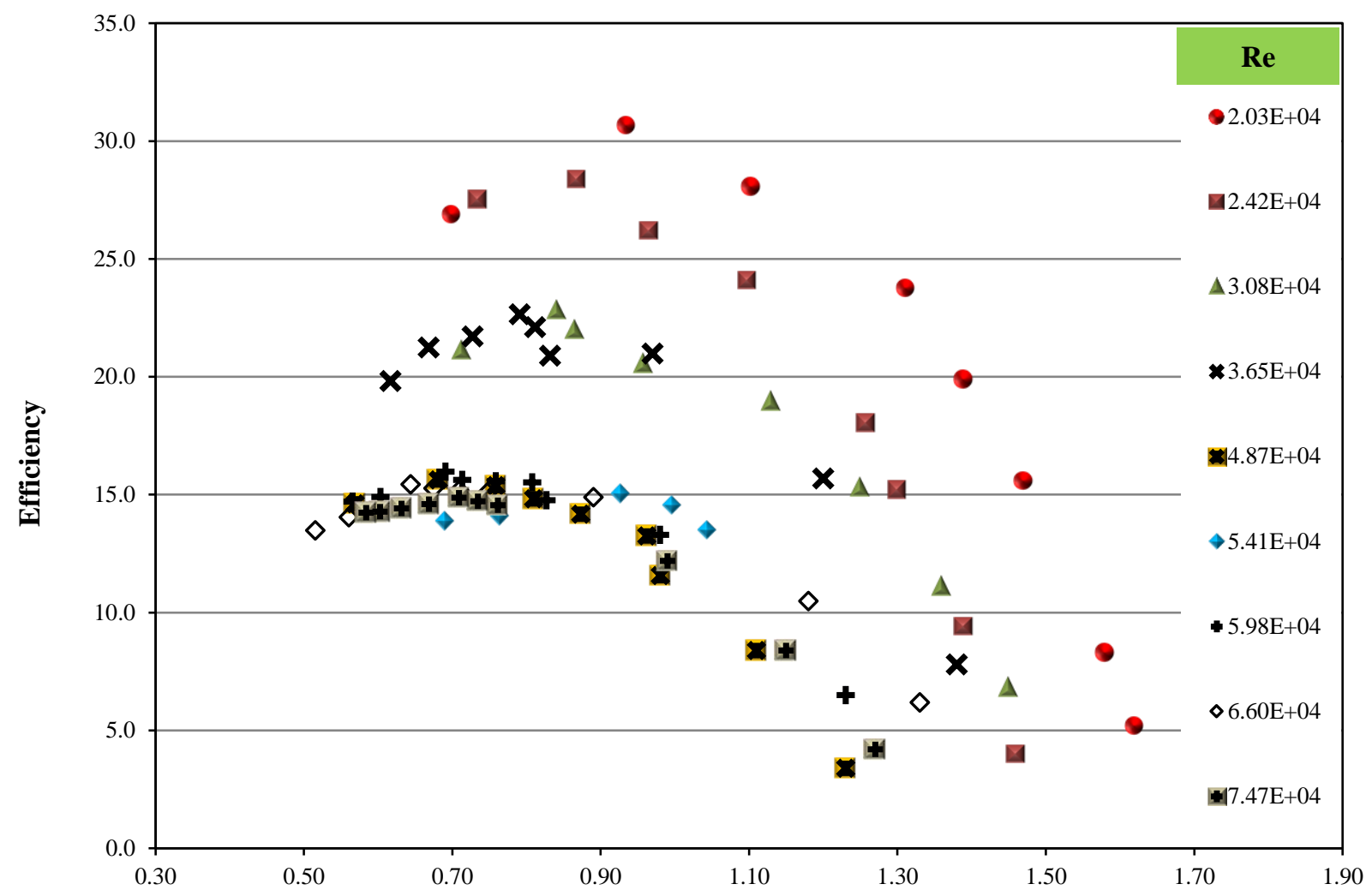

Tip Speed Ratio

Figure 16: Experimental data for efficiency of the VAACT (Flapped Type) in different Reynolds number (I*=0.6). 


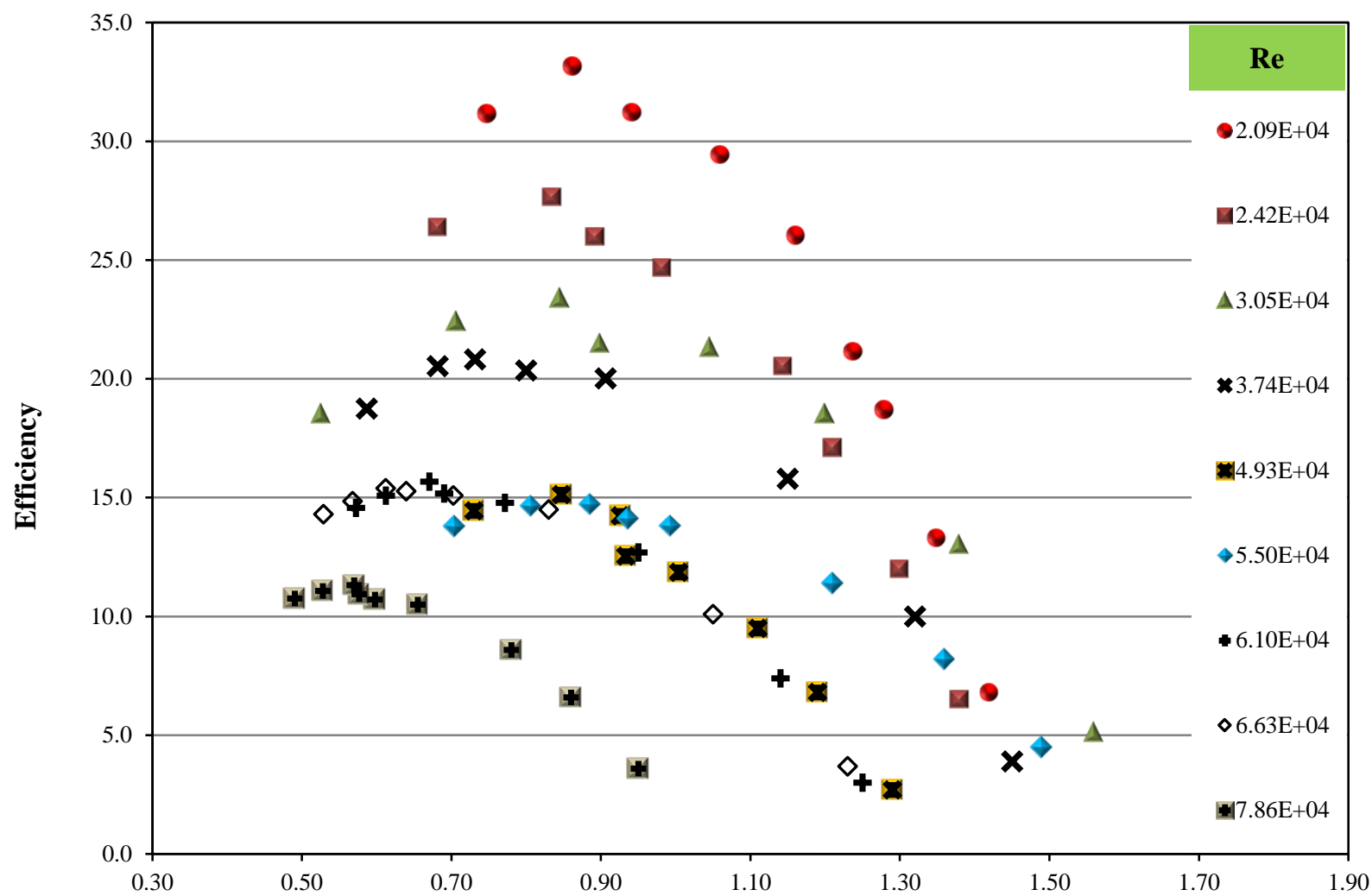

Tip Speed Ratio

Figure 17: Experimental data for efficiency of the VAACT (Flapped Type) in different Reynolds number (I*=0.7). 


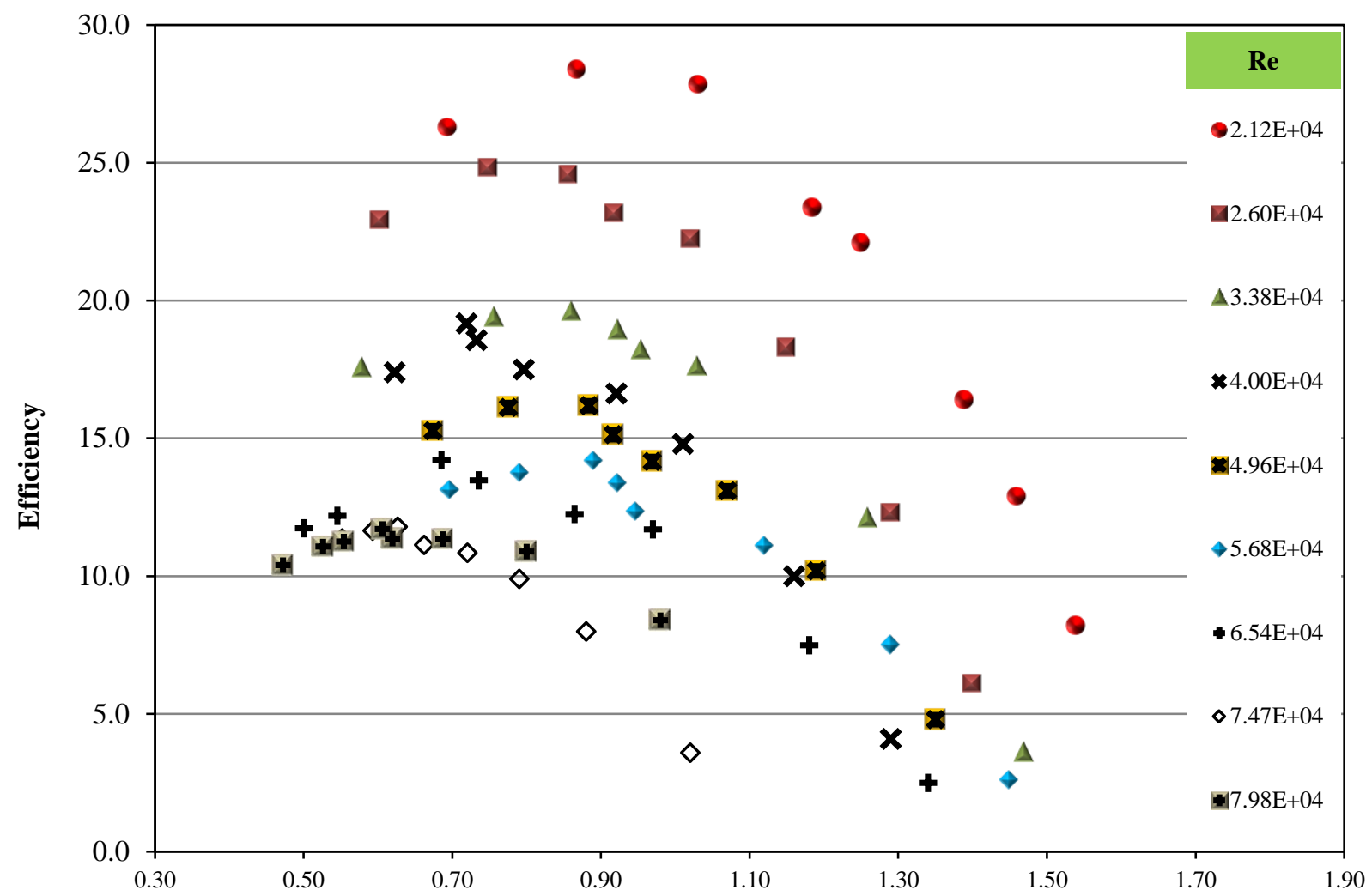

Tip Speed Ratio

Figure 18: Experimental data for efficiency of the VAACT (Flapped Type) in different Reynolds number (I*=0.9).

It can be inferred from the forgoing figures that in each I*, the efficiency of the $\mathrm{S}$ shape type descends by increase in Reynolds number. Except $I^{*}=0.5$, in the other dimensionless mass moments of inertia, the maximum efficiency will take place in the lowest amount of the tested Reynolds numbers. As a rough conclusion, the best range of the operational Reynolds number for this configuration of the VAACT is less than 40000 which its corresponding Froude number is 0.063 . Additionally, it can be concluded by these figures that in the entire Reynolds numbers, the maximum efficiency will occur in the range of 0.6 to 0.7 of the non-dimensional moment of inertia $\left(I^{*}\right)$.

Table 7 gives the maximum power coefficient $\left(\mathrm{Cp}_{\max }\right)$ in each dimensionless moment of inertia (I*). Moreover, Reynolds number, the coefficient of torque, the tip speed ratio and Strouhal number correspond to $\mathrm{Cp}_{\max }$ have been given in this table. Stouhal number of the VAACT match to the best efficiencies varies in range of 0.46 to 0.6 while correspond Reynolds number will change between 20000 and 25000 (congruent channel Froude number between 0.032 and 0.04). For the flapped shape of the vertical axis autorotation current turbine (developed configuration), the efficiency of 33 percent is obtained approximately in the $I^{*}=0.7$.

Table 7: Value of parameters correspond to maximum power coefficient for Flat plate type in different $I^{*}$ 


\begin{tabular}{|c|c|c|c|c|c|c|}
\hline $\begin{array}{c}\text { Dimensionless } \\
\text { moment of inertia } \\
\left(I^{*}\right)\end{array}$ & $\begin{array}{l}\text { Maximum } \\
\text { Coefficient of } \\
\text { performance } \\
\left(C p_{\max }\right) \\
\end{array}$ & $\begin{array}{c}\text { Coefficient of } \\
\text { torque at } \\
\text { Cp }_{\max }\end{array}$ & $\begin{array}{c}\text { Reynolds } \\
\text { Number } \\
(\text { Re) at } \\
\text { Cpmax } \\
\end{array}$ & $\begin{array}{c}\text { Channel } \\
\text { Froude } \\
\text { Number (Fr) } \\
\text { at } \mathrm{Cp}_{\max } \\
\end{array}$ & $\begin{array}{l}\text { Tip speed } \\
\text { ratio } \\
(\lambda) \text { at } \\
\text { Cpmax } \\
\end{array}$ & $\begin{array}{c}\text { Strouhal } \\
\text { Number } \\
\text { (St) at } \\
\text { Cpmax } \\
\end{array}$ \\
\hline 0.4 & 28.7 & 0.335 & 21000 & 0.033 & 0.855 & 0.544 \\
\hline 0.5 & 25.7 & 0.353 & 25000 & 0.04 & 0.727 & 0.463 \\
\hline 0.6 & 30.7 & 0.328 & 20000 & 0.032 & 0.935 & 0.595 \\
\hline 0.7 & 33.2 & 0.385 & 21000 & 0.033 & 0.862 & 0.549 \\
\hline 0.9 & 28.4 & 0.327 & 21000 & 0.033 & 0.868 & 0.553 \\
\hline
\end{tabular}

To probe into the effect of the flaps on the power coefficient of the VAACT, an assessment has been done in the tip speed ratio of 0.65 which places in the optimum range of both configurations (the flat plate and the flapped plate types). Experimental data of the power coefficient match to the tip speed ratio of 0.65 for both configurations have been presented in Table 8 in different Reynolds numbers and I*s. Table 9 presents the ratio of power coefficient of the flapped plate to the flat plate. According to this table, the flap increases the power coefficient significantly. For instance, in the $\mathrm{Re}=60000$ and $\mathrm{I}^{*}=0.9$, flap increases the power coefficient more than five times.

Table 8: Value of power coefficient for Flat plate and S shape type in tip speed ratio of 0.65

\begin{tabular}{c|cc|cc|cc||cc}
\hline \multicolumn{1}{|c|}{ Re } & \multicolumn{2}{|c|}{40000} & \multicolumn{2}{c|}{50000} & \multicolumn{2}{c|}{60000} & \multicolumn{2}{c}{70000} \\
I* & Flat & S & Flat & S & Flat & S & Flat & S \\
\hline \hline 0.4 & 4.5 & 14.5 & 4.5 & 14.3 & 4.4 & 13.6 & 3.5 & 12.9 \\
0.5 & 6.2 & 18.9 & 5.8 & 15.6 & 5.5 & 13.1 & 5.7 & 11.7 \\
0.7 & - & 18.7 & 3.8 & 14.4 & 3.0 & 15.1 & 2.8 & 13.8 \\
0.9 & - & 17.9 & - & 15.1 & 2.4 & 13.2 & 2.8 & 12.5 \\
\hline \hline
\end{tabular}

Table 9: Ratio of power coefficient of S shape type to flat plate type

\begin{tabular}{c|cccc}
\hline I* & $\mathbf{4 0 0 0 0}$ & $\mathbf{5 0 0 0 0}$ & $\mathbf{6 0 0 0 0}$ & $\mathbf{7 0 0 0 0}$ \\
\hline $\mathbf{0 . 4}$ & 3.2 & 3.2 & 3.1 & 3.6 \\
$\mathbf{0 . 5}$ & 3.0 & 2.7 & 2.4 & 2.0 \\
$\mathbf{0 . 7}$ & - & 3.8 & 5.0 & 4.9 \\
$\mathbf{0 . 9}$ & - & - & 5.6 & 4.5 \\
\hline
\end{tabular}

\subsection{Correlation of the performance}

The power coefficient data merge into a single curve by $C_{P} R e^{0.60} / 10^{4} \lambda^{0.5+\sqrt{I^{*}}}$ as shown in Figure 19. A regression analysis was used to predict the correlation equation of this figure. The correlation equation can be expressed by a logarithmic equation which is fitted for the tip speed ratios in the range of 0.45 to 1.55 , the Reynolds number between 20000 and 100000 and the dimensionless moment of inertia ( $\left.I^{*}\right)$ greater than 0.3 . Hence, correlation equation of the flapped plate configuration will be a strip with width of $A$ with respect to the tip speed ratio, viz. 


$$
C_{P} R e^{0.60} / 10^{4} \lambda^{0.5+\sqrt{I^{*}}}=-2.1 \operatorname{Ln}(\lambda)+A \quad\left\{\begin{array}{c}
20000 \leq R e \leq 100000 \\
0.45 \leq \lambda \leq 1.55 \\
I^{*} \geq 0.3
\end{array}\right.
$$

As shown in Figure 19, more than 95 percent of the experimental results of the power coefficient put into the mentioned bandwidth of the A. Parameter A is varied between 0.55 and 1.2 which is dependent on the Reynolds number and the dimensionless moment of inertia (I*). By indication of appropriate value of $\mathrm{A}$, the power coefficient and the torque coefficient $\left(C_{t}=\right.$ $C_{p} / \lambda$ ) will be calculated by means of Eq. 7 with a good accuracy. Figure 20 shows the upper and the lower limit of the correlation curves and their corresponding parameter (A) for different $\mathrm{I}^{*}$.

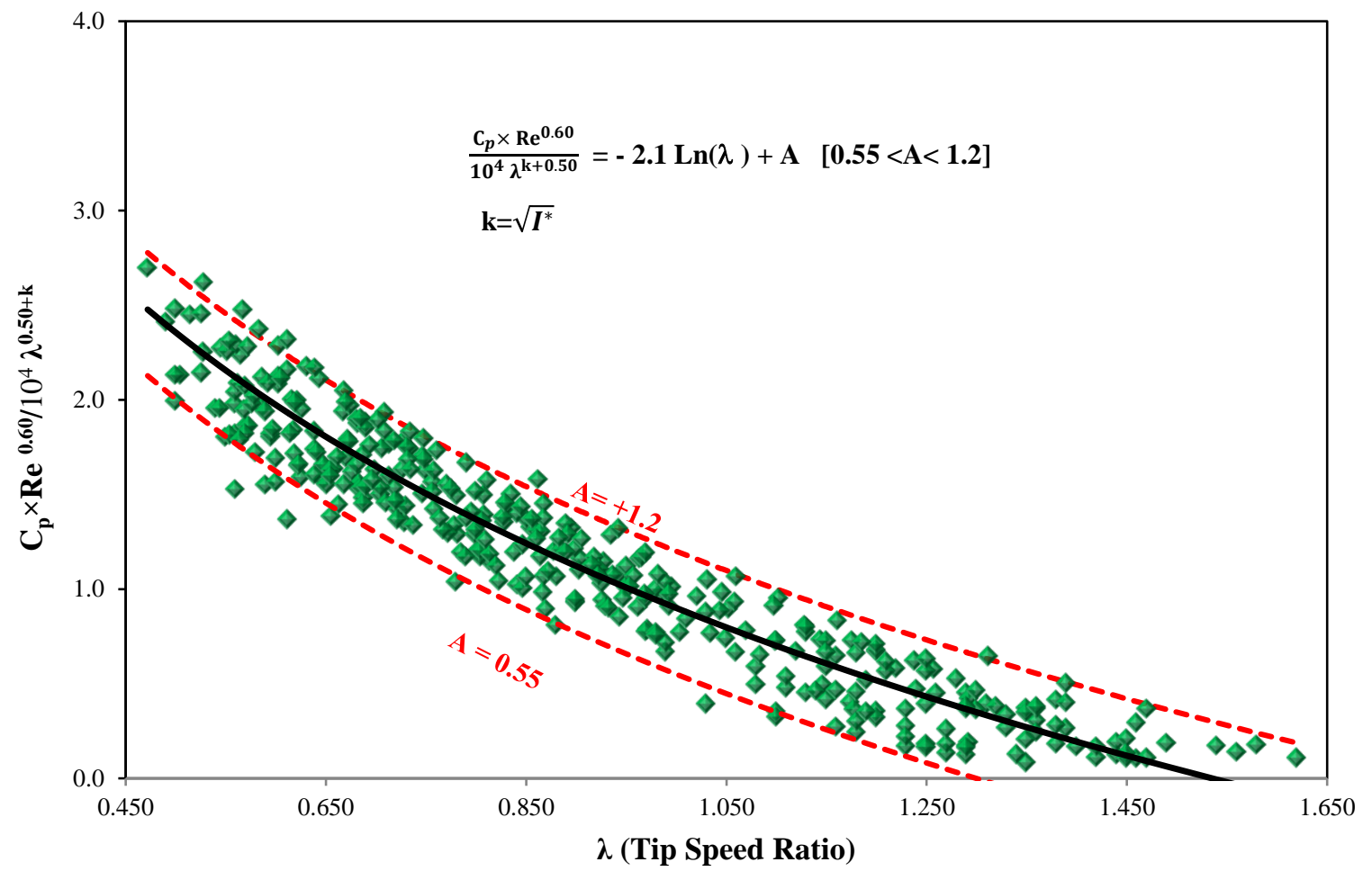

Figure 19: Correlation curve of power coefficient for flat plate type of VAACT. 

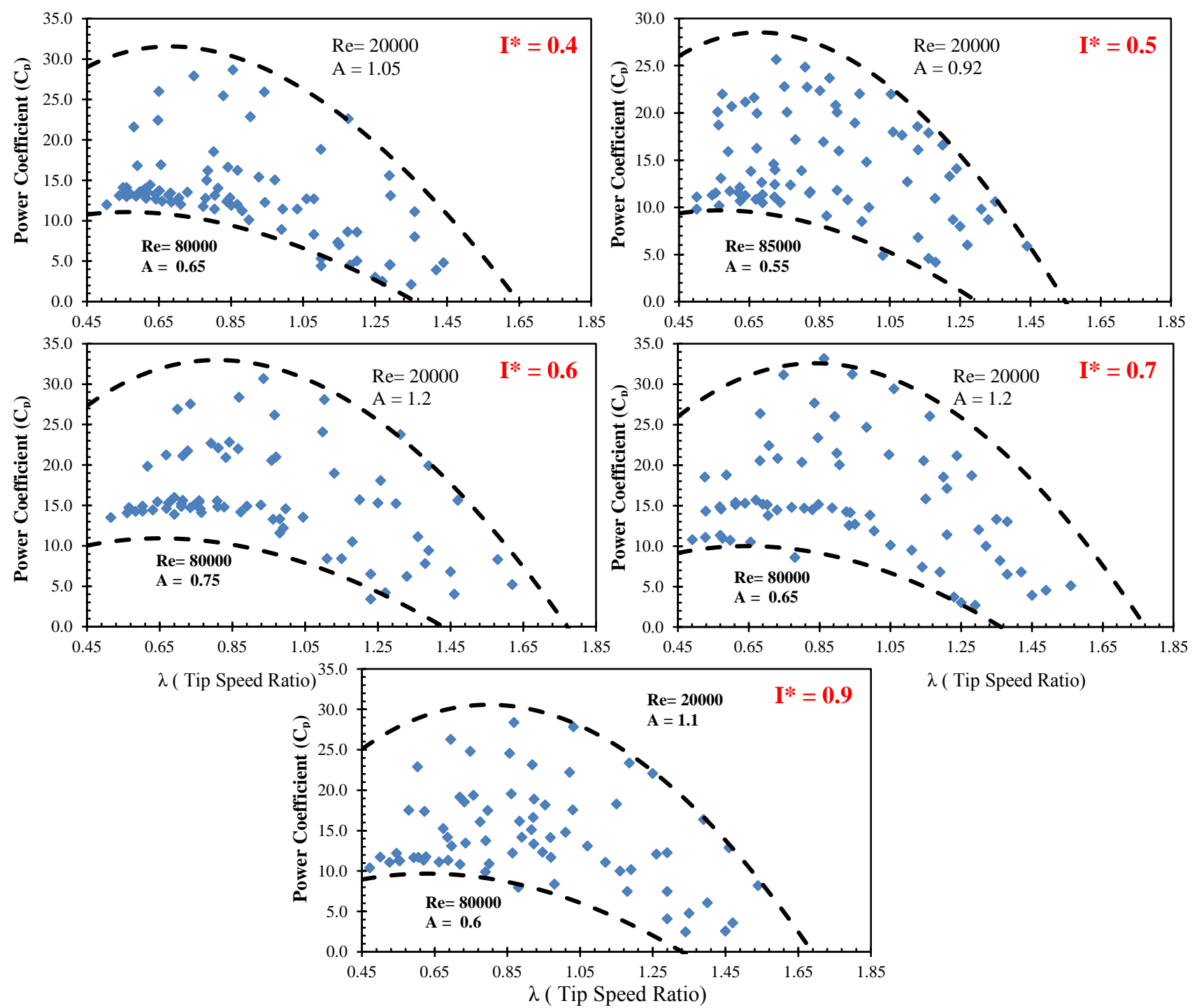

Figure 20: Limitation curves of Correlation of power coefficient and corresponding parameter A for different $\mathrm{I}^{*}$.

\section{Conclusion}

The purpose of the current study was to determine an innovative model of the turbine as a plate which is hinged vertically in the axis of symmetry and exploits the autorotation phenomenon to harvest energy. This model is named Vertical Axis Autorotation Current Turbine (VAACT). This paper devotes to explore experimentally about the flapped plate type or the $\mathrm{S}$ shape type of the VAACT as its developed configuration.

The adequate starting moment and the sufficient mass moment of inertia of the system are two necessary conditions which shall be provided in the VAACT in order to the rotation to be Continuous. Hence, this turbine utilizes the extra mass moment of inertia.

Increase in Reynolds number causes to increase in the angular velocity while, the tip speed ratio goes down. The optimum value of $I^{*}$ correspond to the tip speed ratio and the angular 
velocity is greater than 0.9 . Moreover, the flap increases the angular velocity and the tip speed ratio averagely 60 percent with respect to the flat plate type.

Due to the high dissipation of energy by vortex shedding in higher Reynolds number and consequently drop in the efficiency, the low Reynolds number is more appropriate to operate. As a rough conclusion, the best range of operational Reynolds number for $S$ shape type of the VAACT is less than 40000. In addition, the optimum tip speed ratio for the highest efficiencies places in the range of 0.65 to 0.85 . Furthermore, in the entire Reynolds numbers, maximum efficiency will occur in the range of 0.6 to 0.7 of the non-dimensional moment of inertia (I*).

This study has shown that the flap increases the power coefficient significantly. This study found that the flapped shape of the vertical axis autorotation current turbine can reach to 33 percent of the efficiency in $I^{*}=0.7$ and $\mathrm{Re}=21000$.

\section{Acknowledgment}

The authors express their thanks to ANP (Brazilian National Petroleum Agency), and LOC/COPPE/UFRJ (Laboratory of Waves and Current of COPPE, Federal University of Rio de Janeiro).

\section{Reference}

[1] Khan MJ, Bhuyan G, Iqbal MT, Quaicoe JE. Hydrokinetic energy conversion systems and assessment of horizontal and vertical axis turbines for river and tidal applications A technology status review. Appl. Energy 2009; 83: p. 1823-35.

[2] Rogner H, Barthel F, Cabrera M, Faaij A, Giroux M, Hall D, Kagramanian V, et al. Energy resources. In: World Energy Assessment. Energy and the Challenge of Sustainability" United Nations Development Programmed, United Nations Department of Economic and Social Affairs, World Energy Council, New York, USA: 2000; p. 508.

[3] Rourke FO, Boyle F, Reynolds A. tidal energy update 2009. Applied Energy 2010; 87: p. 398-409.

[4] DTI: Meeting the energy challenge: a white paper on energy. The Stationary Office 2007.

[5] Güney MS, Kaygusuz K. Hydrokinetic energy conversion systems: a technology status review. Renew Sustain Energy Rev 2010; 14: p. 2996-3004.

[6] EPRI. Assessment of Waterpower Potential and Development Needs. Electrical Power Research Institute, Palo Alto, CA, USA: 2007; p. 110.

[7] Mesquita ALA, Palheta FC,Vaz JRP, et al. A methodology for the transient behavior of horizontal axis hydrokinetic turbines. Energy Convers Manage 2014; 87:p. 1261-1268.

[8] Mukherji SS, Kolekar N, Banerjee A, Mishra R. Numerical investigation and evaluation of optimum hydrodynamic performance of a horizontal axis hydrokinetic turbine. Renew Sustainable Energy 2011; 3:063105. 
[9] Vermaak HJ, Kusakana K, Koko SP. Status of micro-hydrokinetic river technology in rural applications: a review of literature. Renewable and Sustainable Energy Review 2014; 29: p. 62533.

[10] Anyi M, Kirke BE. Valuation of small axial flow hydrokinetic turbines for remote communities. Energy for Sustain Develop 2010; 14(2): p. 110-6.

[11] McAdam RA, Houlsby GT, Oldfield MLG. Experimental measurements of the hydrodynamic performance and structural loading of the transverse horizontal axis water turbinePart 1. Renew energy 2013; 59: p. 105-14.

[12] Gorlov AM. Helical turbines for the Gulf Stream: conceptual approach to design of a large scale floating power farm. Marine Technology 1998; 35: p. 175-82.

[13] Khan NI, Iqbal T, Hinchey M, Masek V. PERFORMANCE OF SAVONIUS ROTOR AS A WATER CURRENT TURBINE. Ocean Technology: Maritime and Port Security 2009; 4(2): p.72-83.

[14] Gorban AN, Gorlov AM, Silantyev VM. Limits of the turbine efficiency for free fluid flow. Energy Resous Techno, Trans of the ASME 2001; 123(4): p. 311-7.

[15] Bachant P, Wosnik M. Performance measurements of cylindrical- and spherical-helical cross-flow marine hydrokinetic turbines, with estimates of exergy efficiency. Renew Energy 2015; 74, p. 318-25.

[16] Ramos V, Iglesius G. Performance assessment of tidal stream turbines - a parametric approach. Energy Convers Manage 2013; 69: p. 49-57.

[17] Antheaume S, Matre T, Achard JL. Hydraulic Darrieus turbines efficiency for free fluid flow conditions versus power farms conditions. Renewable Energy 2008; 33: p. 2186-2198.

[18] Kirke BK. Tests on ducted and bare helical and straight blade Darrieus hydrokinetic turbines. Renew energy 2011; 36: p. 3013-22.

[19] Ponta F, Dutt GS. An improved vertical axis water current turbine incorporating a channeling device. Renew Energy 2000; 20(2): p. 223-41.

[20] Malipeddi AR, Chatterjee D. Influence of duct geometry on the performance of Darrieus hydro-turbine. Renew Energy 2012; 43: p. 292-300.

[21] Sarma NK, Biswas A, Misra RD. Experimental and computational evaluation of Savonius hydrokinetic turbine for low velocity condition with comparison to Savonius wind turbine at the same input power. Energy Convers Manage 2014; 83: p. 88-98.

[22] Kyozuka Y. An experimental study on the Darrieus-Savonius turbine for the tidal current power generation. Fluid Science and Technology 2008; 3(3): p. 439-49.

[23] Golecha K, Eldho TI, Prabhu SV. Influence of the deflector plate on the performance of modified Savonius water turbine. Applied Energy 2011; 88: p. 3207-17. 
[24] Moutsoglou A, Weng Y. Performance Tests of a Benesh Wind Turbine Rotor and a Savonius Rotor. wind engineering 1995; 19( 6): p. 349-62.

[25] Yang B, Lawn C. Fluid dynamic performance of a vertical axis turbine for tidal currents. Renewable Energy 2011; 36: p. 3355-3366.

[26] Hwang SI, Lee YH, Kim SJ. Optimization of cycloidal water turbine and the performance improvement by individual blade control. Applied Energy 2009; 86: p. 1532-1540.

[27] Fernandes AC, Armandei M. Low-head hydropower extraction based on torsional galloping. Renew energy 2014; 69: p. 447-52.

[28] Armandei M, Fernandes AC. Marine Current Energy Extraction Using Torsional Galloping. Proceedings of Offshore Technology Conference 2013, Houston, Texas, USA: OTC2013.

[29] Armandei, M, Fernandes AC, Bakhshandeh Rostami A. Hydroelastic Buffeting assessment over a Vertically Hinged Flat Plate. Experimental Techniques 2015; DOI: 10.1111/ext.12134.

[30] Fernandes AC, Bakhshandeh Rostami A, Canzian LG, Mirzaeisefat S. Vertical Axis Current Turbine (VACT) and its Efficiency. Proceedings of the ASME 2013 32nd International Conference on Ocean, Offshore and Arctic Engineering, Nantes France: OMAE 2013; p. V008T09A051: 6 pages.

[31] Fernandes AC, Bakhshandeh rostami A. Hydrokinetic Energy Harvesting by an Innovative Vertical Axis Current Turbine. Accepted to publish in Renewable Energy.

[32] Bakhshandeh rostami A, Fernandes AC. Plate shape effect on the performance of the vertical axis autorotation current turbine (VAACT). Proceedings of the ASME 2014 33nd International Conference on Ocean, Offshore and Arctic Engineering San Francisco, California, USA: OMAE2014; p. V09AT09A041: 8 pages.

[33] Skews BW. Autorotation of many-sided bodies in an airstream. Nature 1991; 352: p.512513.

[34] Ghadimi P, Bakhshandeh Rostami A, Jafarkazemi F. Aerodynamic analysis of the boundary layer region of symmetric airfoils at ground proximity. Aerospace science and technology 2012; 17(1): p. 7-20.

[35] Zeraatgar H, Bakhshandeh Rostami A, Nazari A. A study on performance of planing-wing hybrid craft. Polish Maritime Research 2012; 19(4): p. 16-22.

[36] Iversen JD. Autorotating flat-plate wings: the effect of the moment of inertia, geometry and Reynolds Number. Fluid Mech. 1979; 92: p. 327-348.

[37] Lugt HJ. Autorotation. Annual Review Fluid Mechanics 1983; 15: p.123-147.

[38] Skews BW. Autorotation of rectangular plates. Fluid Mechanics 1990; 217: p. 33-40. 
[39] Bakhshandeh rostami A, Fernandes AC. Simulation of Fluttering and Autorotation motion of Vertically Hinged Flat Plate. Proceedings of the ASME 2015 34nd International Conference on Ocean, Offshore and Arctic Engineering St. John's, NL, Canada: OMAE2015-41244.

[40] Andersen A, Pesavento U, Jane Wang Z. Unsteady aerodynamics of fluttering and tumbling plates. Fluid Mechanics 2005; 541: p. 65-90.

[41] Mirzaeisefat S, Fernandes AC. Stability analysis of the fluttering and autorotation of flowinduced rotation of a hinged flat plate. Hydrodynamic 2013; 25(5): p. 755-62.

[42] Maskel EC. A Theory of the Blockage Effects on Bluff Bodies and stalled wings in a closed wind tunnel. Aeronautical research council: reports and memoranda No 3400: 1963.

[43] Klaka K, Penrose JD, Horsley RR, Renilson MR. Hydrodynamic test on fixed plate in uniform flow. J. experimental in thermal and fluid science 2005; 30: p. 131-39. 